\title{
Modeling glioma invasion with anisotropy- and hypoxia-triggered motility enhancement: from subcellular dynamics to macroscopic PDEs with multiple taxis
}

\author{
G. Corbin, C. Engwer, A. Klar, J. Nieto, J. Soler, C. Surulescu, and M. Wenske
}

June 23, 2020

\begin{abstract}
We deduce a model for glioma invasion making use of DTI data and accounting for the dynamics of brain tissue being actively degraded by tumor cells via excessive acidity production, but also according to the local orientation of tissue fibers. Our approach has a multiscale character: we start with a microscopic description of single cell dynamics including biochemical and/or biophysical effects of the tumor microenvironment, translated on the one hand into cell stress and corresponding forces and on the other hand into receptor binding dynamics; these lead on the mesoscopic level to kinetic equations involving transport terms w.r.t. all kinetic variables and eventually, by appropriate upscaling, to a macroscopic reaction-diffusion equation for glioma density with multiple taxis, coupled to (integro-)differential equations characterizing the evolution of acidity and macro- and mesoscopic tissue. Our approach also allows for a switch between fast and slower moving regimes, according to the local tissue anisotropy. We perform numerical simulations to investigate the behavior of solutions w.r.t. various scenarios of tissue dynamics and the dominance of each of the tactic terms, also suggesting how the model can be used to perform a numerical necrosis-based tumor grading or support radiotherapy planning by dose painting. We also provide a discussion about alternative ways of including cell level environmental influences in such multiscale modeling approach, ultimately leading in the macroscopic limit to (multiple) taxis.
\end{abstract}

\section{Introduction}

Glioma is a common type of primary, fast growing brain tumor with a poor prognosis, the median survival time with the most frequent (and most aggressive) form called glioblastome multiforme amounting at 60 weeks, in spite of modern treatment involving resection, radio-, and chemotherapy (see e.g., 91 and references therein). An exhaustive removal of the tumor is in general impossible, due to the rapid advancement of glioma cells through their cycle and to the diffuse tumor infiltration. This leads to serious clinical challenges and to rather modest treatment outcomes. The correct assessment of tumor margins and of the related GTV, CTV, and PTV ${ }^{1}$ is therefore of utmost importance. Noninvasive medical imaging techniques like MRI and CT are valuable diagnostic tools, however they only provide a macroscopic classification of neoplastic regions and the surounding oedema, without being able to evaluate the actual tumor extent which is heavily influenced by the biological, mechanical, and chemical processes on single cell and subcellular levels. Moreover, every patient has a different brain structure and there is abundant evidence that glioma follow the white matter tracts made up of myelinated axon bundles 32 33]. Therefore, the personalized prediction of the tumor burden is necessary for an enhanced therapy planning and mathematical models are called upon to provide such information via numerical simulations, thereby relying on medical data, of which diffusion tensor imaging (DTI) is quite common 80]. It measures the spatial diffusion of water molecules by MRI per volume element (voxel). The information obtained in this way can be expressed in the form of a diffusion tensor containing the full (apparent) diffusion information along six directions. One way of visualizing

\footnotetext{
${ }^{1} \mathrm{GTV}=$ gross tumor volume; $\mathrm{CTV}=$ clinical target volume; PTV=planning target volume
} 
diffusion tensors is by the so-called fractional anisotropy index FA which is a measure for the local tissue alignment and can directly be obtained from DTI data; it is a scalar value between zero and one, calculated by using the eigenvalues of the apparent diffusion tensor. A value close to one means high anisotropy, i.e., a strong preference for a specific direction, whereas a very small value corresponds to the nearly isotropic case. For more details we refer e.g., to [10], see also 25. For more about DTI and its visualization approaches along with advantages, drawbacks and extensions we refer e.g., to [2,24, 44 .

Several approaches to modeling glioma invasion have been considered so far; they range from discrete formulations $36,45 \mid 46$ ] over hybrid settings $31,82,93]$ combining decriptions of individual cells moving on a lattice with PDEs describing the evolution of some stimulus (e.g., a chemoattractant) and up to pure continuum models coupling several types of differential equations. The latter category comprises in turn several classes of models, according to the scales taken into account and to the type of PDEs employed. Pure macroscopic models using reaction-diffusion equations (possibly with space- and time-dependent diffusion coefficients, or accounting for the interaction with the surrounding tissue by letting the diffusion coefficient be proportional to the water diffusion tensor assessed by DTI) have been introduced e.g., in $13,43,53,85$ to characterize the evolution of glioma density; in 21 some biomechanical effects have been included as well. While these models impose the precise form of the equations and their coefficients, other approaches 39,72 deduce them by using a scaling argument from a mesoscopic setting relying on kinetic transport equations for the glioma density function depending -supplementary to time and position- also on the cell velocities. These models have been further extended in $25,27,41]$ to take into account subcellular level effects on the mesoscopic and ultimately macroscopic behavior of the tumor. Concretely, these multiscale settings included on the microscopic level receptor binding to tissue fibers, which was related to the mesoscale by way of an additional transport term and by the turning rate. Appropriate scalings then led to the macroscale dynamics, whose deduced coefficients are still carrying subcellular level information. In 68 different types of scaling have been considered for this modeling approach, and a well-posedness result was provided for a model also involving the time-space evolution of tissue. A more general analytical result in less regular function spaces for a complex micro-meso-macroscale model involving both chemoand haptotaxis introduced on the mesolevel has been proved in 61 .

Here we propose a multiscale model starting from a kinetic transport equation formulation and accounting, too, for receptor binding dynamics. Unlike in [25 27, 41] where the space-dependent tissue was given by DTI data at a fixed time point and the glioma cells were migrating on this brain structure, in the current model the tissue is allowed to evolve in time as well and the glioma cells degrade it according to their respective orientation to the tissue fibers, similarly to the way introduced in 38, and readdressed in 61, 68. Moreover, in this work we also include a characterization of velocity changes via a transport term involving derivatives with respect to the velocity variable, hence allowing for external forces to act on the cells. Specifically, these external actions are decisively influenced by the spatial gradients of tissue fibers in the tumor surroundings and by the bias induced through the acidity gradient. Reference 19 considered a similar approach accounting for a given chemotactic force acting on the cells and being proportional to the concentration gradient of some non-evolving chemoattractant. Here we pay more detailed attention to the influence of time- and space-dependent acidity and tissue on the cell migration, proliferation, and depletion, thereby also looking into the effect of tissue fiber orientation, hence dealing with both macroscopic and mesoscopic tissue evolution. By formal upscaling we then obtain a system of ordinary and partial (integro-)differential equations with (myopic) diffusion, multiple taxis, and nonlinear source terms, which couples macroscopic dynamics of tumor cells, necrotic matter, acidity, and normal tissue with that of mesoscopic tissue. The rest of the paper is organized as follows: in Section 2 we set up the micro- and mesoscopic descriptions of (sub)cellular glioma dynamics in the kinetic theory of active particles (KTAP) framework developed e.g., in 66,7 and also provide descriptions of the other involved model variables: tissue, necrotic matter, and acidity. Section 3 is dedicated to deducing effective equations for the macroscopic glioma density influenced by tissue and acidity dynamics. Formal parabolic and hyporbolic upscalings are performed and a low-order moment approximation is deduced for the characterization of mesoscopic tissue, in order to reduce the complexity of the system. Numerical simulations are conducted in Section 4 for three different scenarios of increasing complexity, and the obtained results are compared and commented. Finally, Section 5 provides a discussion of this work's outcome, model extensions, and perspectives, along with a review of various ways of multiscale modeling of tactic cell behavior in the KTAP framework. 


\section{Set up of the micro-meso model}

Our modeling approach aims at obtaining a macroscopic description of the tumor cell evolution in interaction with the underlying tissue. The resulting PDE should carry in its coefficients information about relevant processes occuring on the lower levels (subcellular/microscopic, individual cells/mesoscopic) and should be deduced from mathematical descriptions of the dynamics on the latter. On the macroscopic scale the two main components of cell motion in a tissue should be retrieved, namely diffusion and haptotaxis.

We start by introducing some notations for the various variables and functions involved in this work.

\section{Notation 1}

- Mechanical variables: time $t \in \mathbb{R}^{+}$, space $x \in \mathbb{R}^{N}$, velocity $v \in V:=s \mathbb{S}^{N-1}$, where $\mathbb{S}^{N-1}$ is the unit sphere in $\mathbb{R}^{N}$, and, as in $25,27,38,42$, we assume the cell speed $s>0$ to be constant.

- $\hat{v}:=\frac{v}{|v|}$ a unitary vector denoting the direction of a vector $v \in V$.

- $Q(t, x)$ : macroscopic tissue density.

- Biological (activity) variable y: volume fraction of bound receptors on the cell membrane. Its dynamics is given by an ODE obtained for $y$ by mass action kinetics of receptor bindings and detachments (with rates $k_{+}$and $k_{-}$, respectively):

$$
\dot{y}=k_{+}\left(R_{0}-y\right) Q(t, x)-k_{-} y, \quad y \in\left(0, R_{0}\right) \text {. }
$$

This represents in our model the subcellular dynamics. Thereby, as in previous works [49 68, we assume for simplicity that the total amount of cell membrane receptors $R_{0}$ is constant and we rescale $y \rightarrow y / R_{0}$, thus obtaining ${ }^{2}$

$$
\dot{y}=G(y, Q):=k_{+}(1-y) Q(t, x)-k_{-} y, \quad y \in Y:=(0,1)
$$

- $p(t, x, v, y)$ : density function of tumor cells (mesoscopic tumor cell density). We will also use $\bar{p}(t, x, y):=\int_{V} p(t, x, v, y) d v$.

- $\rho(t, x):=\int_{Y} \bar{p}(t, x, y) d y=\int_{Y} \int_{V} p(t, x, v, y) d v d y$ : macroscopic density of tumor cells.

- $q(t, x, \theta)$ : directional distribution function of tissue fibers with orientation $\theta \in \mathbb{S}^{N-1}$, where $\mathbb{S}^{N-1}$ denotes the unit sphere in $\mathbb{R}^{N}$. Hence $\int_{\mathbb{S}^{N-1}} q(t, x, \theta) d \theta=1$.

- $\mathbb{E}_{q}(t, x):=\int_{\mathbb{S}^{N-1}} \theta q(t, x, \theta) d \theta$ : mean fiber orientation.

- $\mathbb{V}_{q}(t, x):=\int_{\mathbb{S}^{N-1}}\left(\theta-\mathbb{E}_{q}(t, x)\right) \otimes\left(\theta-\mathbb{E}_{q}(t, x)\right) q(t, x, \theta) d \theta$ : variance-covariance matrix for orientation distribution of tissue fibers.

- $h(t, x)$ : concentration of acidity (protons), a genuinely macroscopic quantity.

- $n(t, x)$ : density of necrotic tissue (also a macroscopic quantity).

- $\lambda(x, y)$ : cell turning rate.

Next we characterize innovations of cell density function due to changes in velocity and activity variable under the influence of the tissue structure. Depending on their nature, these are incorporated into the model in different ways:

(i) As transport parts of the mesoscopic equation for the cell density function $p$ : a $\operatorname{div}_{y}$ term accounts for the binding of cell receptors to tissue fibers, while a $\operatorname{div}_{v}$ term describes biomechanical effects, such as cell stress and directionality dictated by the spatial variations in (mesoscopic) tissue density and macroscopic acidity concentration.

(ii) As a jump process, being modeled as an integral operator $L_{0}$ on the right-hand side of the equation. The kernel of that operator will depend as in previous works $25,27,38,42$ on the tissue structure, by way of the fiber directional distribution $q(t, x, \hat{v})$, as will be explained below.

\footnotetext{
${ }^{2}$ Observe that the activity variable $y$ belongs to an open set; indeed, $y=0$ would mean that no receptors are bound (which is not possible for cells living in tissue), while $y=1$ would correspond to all receptors being occupied (which actually never happens, as it would impede migration, proliferation, and even cell survival, due to anoikis). For an actual mathematical justification we refer to 49.61 .
} 
(iii) As a source term describing proliferation/decay and being modeled as another integral operator $\mathcal{P}$. This can be done for instance similarly to the approach in [26, where the interaction of cells with the surrounding tissue was considered to be at the onset of cell survival and mitosis. Here we modify that approach in order to account for the unfavorable effect of acidity on these processes. The concrete form of the corresponding term $\mathcal{P}(p, Q, h, \rho)$ will be specified later.

Thus, the equation for $p$ takes the following form:

$$
\begin{aligned}
\partial_{t} p+v \cdot \nabla_{x} p+\partial_{y}(G(y, Q) p) & +\alpha \operatorname{div}_{v}\left(\mathbb{B}(v)\left(\nabla_{x} q(t, x, \hat{v})-\nabla_{x} h(t, x)\right) p\right) \\
& =\lambda(x, y) L_{0}(p)+\mathcal{P}(p, Q, h, \rho),
\end{aligned}
$$

where we used the notation $\mathbb{B}(v):=|v|^{2} \mathbb{I}_{N}-v \otimes v$, and where the transport coefficients are obtained from the subcellular (microlevel) dynamics 3

$$
\frac{d x}{d t}=v ; \quad \frac{d v}{d t}=\alpha \mathbb{B}(v)\left(\nabla_{x} q(t, x, \hat{v})-\nabla_{x} h(t, x)\right) ; \quad \frac{d y}{d t}=G(y, Q),
$$

the latter equation in (2.4) being just 2.1) and the middle equation characterizing the dynamics of $v$ influenced by the space gradients of the directional distribution of tissue fibers and of the acidity. Thereby, the tensor $v \otimes v$ represents the active cell stress, while $-|v|^{2} \mathbb{I}_{N}$ is the isotropic part. Indeed, concerning cell velocities we are foremost paying attention to the direction of the vector $v$ and consider that it is mainly influenced by the fiber distribution (and in particular by its spatial variations), as well as by the acidity gradient. The latter is known to drive the tumor cells and seems to have a bidirectional effect: Low extracellular $\mathrm{pH}$ favorizes both their migration and proliferation [29 79 86], but acidosis can also inhibit cell proliferation, induce stress response, and apoptosis, see e.g. [69]. Specifically in glioblastoma multiforme (GBM) it can trigger motion in the opposite direction of the acidity gradient. Indeed, GBM cells form typical, garland-like structures called pseudopalisades, which are centered around the highly hypoxic occlusion site of a capillary [14 89. The presence of such histological patterns is an indication for poor prognosis of patient survival 51. In order not to complicate the exposition too much we will only consider here the latter situation, with the repellent effect of acidity, hence the motion of glioma cells being biased towards $-\nabla_{x} h$.

The dimensionless parameter $\alpha$ will play an important role, since it incorporates information from the different scales on which the tumor density, fiber directional distribution or acidity are correlated. While the tumor has volume dimensions, the path of travel made up of targeting fibers has a thinner structure close to the flat one. We can assume that the relationship with acidity is similar in scale. Therefore, the gradients of $q$ and $h$ w.r.t. $x$ contribute with this new scale parameter $\alpha$ among the agents of this process, which must be, as a consequence, a large quantity.

Observe that the dynamics of $v$ in (2.4) ensures that $\frac{d|v|^{2}}{d t}$, hence the cell speed $|v|=s$ is constant (in line with the above assumption $V=s \mathbb{S}^{N-1}$ ), hence only the direction matters.

For the evolution of acidity concentration $h$ we also need to provide an equation; it will take the form of a reaction-diffusion PDE describing the facts that protons diffuse through the entire available space with diffusion parameter $D_{H}$, acidity is produced by cancer cells, and may infer decay, e.g. through uptake by vasculature or normal cells. We denote by $b>0$ the uptake rate and consider a limited production by cancer cells irrespective of their motility and/or receptor binding state, with a constant rate $a>0$. In 2.5p the constant $h_{0}$ denotes an acidity threshold which is critical for non-cancerous matter; the proton buffering is enhanced when that threshold is exceeded, and it is limited by the availability of normal tissue and cells, which decreases under hypoxia (as explained later on):

$$
h_{t}=D_{H} \Delta h+\frac{a \rho}{1+\rho}-b Q\left(h-h_{0}\right) .
$$

The turning operator $L_{0}$ on the right hand side in $(2.3)$ is taken as a relaxation-type operator describing the velocity-jump discontinuities in the cell density function $p$ due to contact guidance,

\footnotetext{
${ }^{3}$ Strictly speaking we understand the ODEs for $v$ and $y$ as being written for tissue $q, Q$ and acidity $h$, scaled with their reference quantities (e.g., tissue carrying capacity $K_{Q}$ and acidity threshold $h_{0}$ introduced below), but for the sake of simplifying the notation we do not explicitly write those denominators. Same applies to the complementary solution components involved in the source terms of the forthcoming equations for cell density, acidity, and necrotic matter.
} 
that is, these velocity alterations are assumed to be caused by the cells interacting with tissue fibers and adapting their motion to biochemical (biomechanical, etc.) cues available at sites in their direct proximity. More precisely, it is given by

$$
L_{0}(p):=\bar{p}(t, x, y) M(t, x, v)-p(t, x, v, y)
$$

where $M(t, x, v)$ depends on the fiber directional distribution $q$ :

$$
M(t, x, v):=\frac{q(t, x, \hat{v})}{\omega}, \quad \omega:=\int_{V} q(t, x, \hat{v}) \mathrm{d} v
$$

thus $\int_{V} M(t, x, v) \mathrm{d} v=1$. Notice that this corresponds to considering, as in 25, 27, 38, 72, a turning operator of type

$$
L_{0}(p):=-p(t, x, v, y)+\int_{V} \tilde{K}\left(v, v^{\prime}\right) p\left(t, x, v^{\prime}, y\right) d v^{\prime}
$$

with the turning kernel defined by $\tilde{K}\left(v, v^{\prime}\right)=\frac{q(t, x, \hat{v})}{\omega}$, that is, the random migration from any velocity $v^{\prime}$ to a new one $v$ is given by the (normalized) orientational distribution of tissue fibers on this last velocity $v$, so through contact guidance. We recall that this turning operator is multiplied by the cell turning rate $\lambda(x, y)$. It will play an essential role in our attempt to characterize the alternation between slow and enhanced migration of tumor cells, which is mainly due to the tissue anisotropy. To capture this dependence we use the fractional anisotropy index FA mentioned in Section 1. Henceforth we deal with a turning rate of the form

$$
\lambda(x, y)=\frac{\kappa y}{F A(x)+y},
$$

which is in agreement with the assumption of reduced turning in highly aligned regions and with its dependence on the receptor binding state $y$, the latter with a certain saturation modeled by the Monod type factor. Thereby, $\kappa$ is a constant that refers to the maximum turning frequency.

Finally, we introduce the proliferation/decay operator. Similarly to the approach in [26], both proliferation and decay of tumor cells originate (at least partially) in the interaction of cells with the surrounding tissue, but here we modify that approach in order to account for the unfavorable effects of acidity on these processes. Concretely, $\mathcal{P}(p, Q, h, \rho)$ is given by

$$
\mathcal{P}(p, Q, h, \rho):=\mu(\rho, h) \int_{Z} \chi\left(x, z, z^{\prime}\right) p\left(t, x, v, z^{\prime}\right) Q(t, x) d z^{\prime},
$$

where $\chi\left(x, z, z^{\prime}\right)$ is a kernel with respect to $z$ and represents the transition from the state $z^{\prime}$ to the state $z$ during a proliferation-favorable glioma-tissue interaction.

For the tissue dynamics we adopt the approach in [38] which was also employed in $49,61,68]$, with slight modifications. We look for the evolution of $q(t, x, \theta)$, hence want an equation of the form

$$
\partial_{t} q(t, x, \theta)=\tau(\theta, p, q),
$$

with $\tau$ satisfying the natural conditions:

$$
\tau(\cdot, \cdot, 0)=0, \quad \int_{\mathbb{S}^{N-1}} \tau(\theta, \cdot, \cdot) d \theta=0, \quad \forall \theta \in \mathbb{S}^{N-1}
$$

We thus compare -as in [38]- the fiber orientation $\theta$ with the cell direction $\hat{v}$, and introduce the operator:

$$
\Pi[p](t, x, \theta):= \begin{cases}\frac{1}{\rho(t, x)} \int_{Y} \int_{V}|\theta \cdot \hat{v}| p(t, x, v, y) \mathrm{d} v \mathrm{~d} y, & \text { for undirected tissue } \\ \frac{1}{\rho(t, x)} \int_{Y} \int_{V} \theta \cdot \hat{v} p(t, x, v, y) \mathrm{d} v \mathrm{~d} y, & \text { for directed tissue }\end{cases}
$$

which averages the projection of cell movement direction on the fiber orientation. It models the fact that cells preferentially degrade fibers which are nearly orthogonal to their movement direction (for more details see [38]). It holds that $0 \leq \Pi[p] \leq 1$ and $-1 \leq \Pi[p] \leq 1$ for undirected and for directed tissue, respectively. Thereby, undirected means that the fibers making up the tissue are symmetrical all along their axes, i.e. there is no 'up' and 'down' on such fibers, which translates into symmetry of the orientational distribution:

$$
q(t, x,-\theta)=q(t, x, \theta), \quad \forall \theta \in \mathbb{S}^{N-1} .
$$


If this is not the case, then the tissue is said to be directed. These tissue properties reflect on the cell motility: While undirected fibers do not impose any supplementary bias on the (re)orientation of a cell, in a directed tissue the cells will have a preferred direction of advancement along the fibers. As DTI data do not provide any information about such directionality, we will consider in the following both types of tissue, which will lead to two different ways of performing the transition from the lower scales (micro and meso) to the macroscopic description of tumor dynamics in interaction with their fibrous and biochemical surroundings.

If we denote by $g(t, x, \theta)$ the mesoscopic density of tissue fibers with orientation $\theta$ we obtain for the evolution of this quantity the equation

$$
\partial_{t} g(t, x, \theta)=r_{D}(h)(\Pi[p](t, x, \theta)-1) \rho(t, x) g(t, x, \theta),
$$

where $r_{D}$ is a nonnegative quantity characterizing the efficiency of fiber degradation (e.g., it can be considered to be proportional to the amount of matrix degrading enzymes 4 expressed by the cells, thus to the acidity $h$, since MDEs are actually known to be enhanced by an acidic extracellular $\mathrm{pH}$, see [16,47]). We then consider $r_{D}$ to be a monotonically increasing function of $h$ with $r_{D}(h)=r_{0}\left(h-h_{0}\right)_{+}$.

It would be important to have information about the directional distribution function $q(t, x, \theta)$, hence we express the latter with respect to the mesoscopic tissue density $g(t, x, \theta)$. Following [38], this relationship takes the form

$$
q(t, x, \theta)=\frac{g(t, x, \theta)}{\int_{\mathbb{S}^{N-1}} g(t, x, \theta) d \theta}, \quad g \neq 0 .
$$

The macroscopic tissue density $Q(t, x)$ represents the volume fraction of tissue fibers, irrespective of their orientation, thus we also have the following relationship:

$$
g(t, x, \theta)=q(t, x, \theta) Q(t, x), \quad t>0, x \in \mathbb{R}^{N}, \theta \in \mathbb{S}^{N-1} .
$$

Integrating 2.9 w.r.t. $\theta \in \mathbb{S}^{N-1}$ we get

$$
\partial_{t} Q=r_{D}(h) \rho Q\left(\int_{\mathbb{S}^{N-1}} \Pi[p](t, x, \theta) q(t, x, \theta) d \theta-1\right)=r_{D}(h) \rho Q \int_{\mathbb{S}^{N-1}}(\Pi[p]-1) q d \theta .
$$

In the sequel the average of $\Pi[p]$ w.r.t. the distribution of fiber orientations will be denoted as in 38] by

$$
A[p](t, x):=\int_{\mathbb{S}^{N-1}} \Pi[p](t, x, \theta) q(t, x, \theta) d \theta,
$$

hence 2.11) becomes

$$
\partial_{t} Q=r_{D}(h) \rho Q(A[p]-1) .
$$

Likewise, we can deduce an equation for the fiber directional distribution function:

$$
\partial_{t} q(t, x, \theta)=r_{D}(h) \rho(t, x) q(t, x, \theta)(\Pi[p](t, x, \theta)-A[p](t, x)) .
$$

Eventually, the decay of both tissue and glioma (primarily due to acidity) generates necrotic tissue, large amounts of which are an indicative of poor survival prognosis [35, 62. Therefore, the detection and assessment of necrosis is an important issue in therapy. Here we describe the dynamics of necrotic tissue density by

$$
\partial_{t} n=r_{D}(h) \rho Q \int_{\mathbb{S}^{N-1}}(1-\Pi[p](t, x, \theta)) q(t, x, \theta) d \theta+\mathcal{F}(h, \rho, Q)
$$

where $\mathcal{F}(h, \rho, Q)$ describes glioma death due to hypoxia and is to be specified later.

Thus, the full model for cell-tissue interactions and response to acidity is given by [2.3], (2.5), 2.13, 2.14, 2.15, supplemented with appropriate initial and boundary conditions.

As in previous works $25,27,49$ we assume $p$ to be compactly supported in the $(v, y)$-space. The initial conditions and the boundary conditions w.r.t. space will be addressed in the following sections. The wellposedness of this problem (especially in less regular function spaces) is not trivial and also not the aim of this paper. When the space domain is the whole $\mathbb{R}^{N}$ the approach in $48,49,61$ could be used as a starting point in order to address this issue.

\footnotetext{
${ }^{4}$ shortly MDEs
} 


\section{Towards population behavior: Upscaling}

Our objective is to assess the macroscopic evolution of the tumor cell population interacting with the brain tissue. As the latter exhibits anisotropy variability, with highly aligned regions alternating with areas of isotropic fiber distribution, the invasive behavior of glioma cells will be correspondingly -and locally- dominated by diffusion with or without drift. Therefore, it is desirable that our model includes a switch between these two kinds of motion, in the sense that the influence of drift together with diffusion can be potentiated against pure diffusion. These effects are built in via the turning rate $\lambda(x, y)$ given in Section 2 above. Indeed, its dependence on the (local) fractional anisotropy $F A(x)$ and the amount of receptors bound to their ligands on the tissue fibers will be able to capture the alternation between the epochs of higher- or less-aligned tissue.

1. Mass conservation: $\int_{V} L_{0}(p)(t, x, v, y) \mathrm{d} v=0$.

2. Self-adjointness: $L_{0}$ is self-adjoint in $L^{2}\left(\frac{d v}{M(v)}\right)$.

3. Kernel of $L_{0}: L_{0}(p)=0 \Leftrightarrow p \in\langle M(v)\rangle$, thus $\operatorname{Ker}\left(L_{0}\right)=\langle M(v)\rangle$, the space generated by $M(v)$. In particular, using the self-adjointness, we know that $M^{\perp} \in\langle M(v)\rangle^{\perp}$ iff $\int_{V} M^{\perp}(v) d v=0$.

Before proceeding with the scaling we re-express (similarly to e.g. 25]) the subcellular dynamics in a more convenient way: Let us consider $y^{*}=f(Q):=\frac{k_{+} \bar{Q}}{k_{-}+k_{+} Q}$, the steady-state of (2.1), and denote by

$$
z:=y-y^{*}
$$

the deviation of the current activity variable $y$ from the steady state. Then $z \in Z \subseteq\left[-y^{*}, 1-y^{*}\right]$ and the microscopic dynamics (2.4) turns into

$$
\frac{d x}{d t}=v ; \quad \frac{d v}{d t}=\alpha \mathbb{B}(v)\left(\nabla_{x} q(t, x, \hat{v})-\nabla_{x} h(t, x)\right) ; \quad \frac{d z}{d t}=-\left(k_{+} Q+k_{-}\right) z-f^{\prime}(Q)\left(Q_{t}+v \cdot \nabla_{x} Q\right) .
$$

Then 2.3 becomes

$$
\begin{aligned}
\partial_{t} p & +v \cdot \nabla_{x} p-\partial_{z}\left(\left(\left(k_{+} Q+k_{-}\right) z+f^{\prime}(Q)\left(Q_{t}+v \cdot \nabla Q\right)\right) p\right)+\alpha \operatorname{div} v\left(\mathbb{B}(v)\left(\nabla_{x} q(\hat{v})-\nabla_{x} h\right) p\right) \\
& =\lambda(x, z) L_{0}(p)+\mathcal{P}(p, Q, h, \rho),
\end{aligned}
$$

where the turning rate in terms of $z$ is given by $\lambda(x, z)=\frac{\kappa(z+f(Q(t, x)))}{F A+z+f(Q(t, x))}$. To simplify the subsequent computations we linearize it as follows:

$$
\lambda(t, x, z) \simeq \lambda_{0}(t, x)+\lambda_{1}(t, x) z, \text { where } \lambda_{0}(t, x)=\frac{\kappa f(Q)}{F A+f(Q)} \text { and } \lambda_{1}(t, x)=\frac{\kappa F A}{(F A+f(Q))^{2}} .
$$

Remark 3.1 Notice that the fractional anisotropy FA changes dynamically, i.e. depend both on $t$ and $x$, since tissue evolution (here this means degradation) leads to local modifications of the water diffusion tensor and, correspondingly, of its eigenvalues. Indeed, the apparent diffusion tensor can even vanish locally, in which situation the cell diffusivity degenerates. In hitherto works $22,23,25,27,41,43,72$ FA has been assumed to be time-independent, motivating that the resolution of DTI data does rarely go below voxels with sizes of $1 \mathrm{~mm}^{3}$, which is rather rough compared with the size of glioma cell bodies $(15-60 \mu m,[56])$. This simplifies the numerical handling and also avoids problems related to the possibly singular behavior of solutions to the macroscopic PDE, as systems with degenerating myopic diffusion and haptotaxis can lead to blow-up even in 1D 87, 88. In Section 4, however, we will consider several simulation scenarios, including, in turn, evolving and time-stationary tissue, and compare their outcome.

\subsection{Parabolic limit, undirected tissue}

The parabolic scaling corresponds formally to the change of variables $t \rightarrow \varepsilon^{2} t, x \rightarrow \varepsilon x$. We perform it and thereby rescale as in 26 the source term $\mathcal{P}(p, Q, h, \rho)$ with $\varepsilon^{2}$, in order to let it act on the correct time scale. Correspondingly, equation 3.16 becomes:

$$
\begin{aligned}
\varepsilon^{2} \partial_{t} p+ & \varepsilon \nabla_{x} \cdot(v p)-\partial_{z}\left(\left(\left(k_{+} Q+k_{-}\right) z+f^{\prime}(Q)\left(\varepsilon^{2} Q_{t}+\varepsilon v \cdot \nabla Q\right)\right) p\right)+\alpha \varepsilon \operatorname{div}_{v}\left(\mathbb{B}(v)\left(\nabla_{x} q(\hat{v})-\nabla_{x} h\right) p\right) \\
& =\lambda(t, x, z) L_{0}(p)+\varepsilon^{2} \mu(\rho, h) Q(t, x) \int_{Z} \chi\left(x, z, z^{\prime}\right) p\left(t, x, v, z^{\prime}\right) d z^{\prime}
\end{aligned}
$$


Next we consider the moments of $p$ with respect to $v$ and especially with $z$ :

$$
\begin{aligned}
& m(t, x, v):=\int_{Z} p(t, x, v, z) d z, \quad m^{z}(t, x, v):=\int_{Z} z p(t, x, v, z) d z, \quad \rho^{z}(t, x)=\int_{V} m^{z}(t, x, v) d v \\
& \bar{p}(t, x, z):=\int_{V} p(t, x, v, z) d v, \quad \rho(t, x):=\int_{V} m(t, x, v) d v=\int_{Z} \bar{p}(t, x, z) d z
\end{aligned}
$$

and neglect the higher order moments with respect to $z$ by assuming very small deviations of the receptor binding dynamics from the steady-state, i.e. by assuming $z$ to be very small. As the subcellular dynamics is very fast in comparison to cell motion and proliferation, this is a reasonable assumption.

Then from 3.17) we obtain the moment equations:

$$
\begin{aligned}
& \varepsilon^{2} \partial_{t} m+\varepsilon \nabla_{x} \cdot(v m)+\alpha \varepsilon \operatorname{div}_{v}\left(\mathbb{B}(v)\left(\nabla_{x} q(\hat{v})-\nabla_{x} h\right) m\right)=\lambda_{0}(t, x)(M(v) \rho-m) \\
& \quad+\lambda_{1}(t, x)\left(M(v) \rho^{z}-m^{z}\right)+\varepsilon^{2} \mu(\rho, h) Q(t, x) \int_{Z} \int_{Z} \chi\left(x, z, z^{\prime}\right) p\left(t, x, v, z^{\prime}\right) d z^{\prime} d z \\
& \varepsilon^{2} \partial_{t} m^{z}+\varepsilon \nabla_{x} \cdot\left(v m^{z}\right)+\alpha \varepsilon \operatorname{div}_{v}\left(\mathbb{B}(v)\left(\nabla_{x} q(\hat{v})-\nabla_{x} h\right) m^{z}\right)+\left(k_{+} Q+k_{-}\right) m^{z}+\varepsilon f^{\prime}(Q) v \cdot \nabla Q m \\
& +\varepsilon^{2} f^{\prime}(Q) m Q_{t}=\lambda_{0}(t, x)\left(M(v) \rho^{z}-m^{z}\right)+\varepsilon^{2} \mu(\rho, h) Q(t, x) \int_{Z} \int_{Z} z \chi\left(x, z, z^{\prime}\right) p\left(t, x, v, z^{\prime}\right) d z^{\prime} d z
\end{aligned}
$$

Performing the usual Hilbert expansion $p=\sum_{k} \varepsilon^{k} p_{k}$ and consequently the expansion of the moments:

$$
m=\sum_{k} \varepsilon^{k} m_{k}, \quad m^{z}=\sum_{k} \varepsilon^{k} m_{k}^{z}, \quad \rho=\sum_{k} \varepsilon^{k} \rho_{k}, \quad \rho^{z}=\sum_{k} \varepsilon^{k} \rho_{k}^{z}
$$

and identifying the powers of $\varepsilon$ we obtain from $(3.18 \mathrm{a})$ and $3.18 \mathrm{~b}$ the following relationships:

$\epsilon^{0}$ terms:

$0=\lambda_{0}\left(M(v) \rho_{0}-m_{0}\right)+\lambda_{1}\left(M(v) \rho_{0}^{z}-m_{0}^{z}\right)$

$\left(k_{+} Q+k_{-}\right) m_{0}^{z}=\lambda_{0}\left(M(v) \rho_{0}^{z}-m_{0}^{z}\right) \quad \Rightarrow \quad \rho_{0}^{z}=m_{0}^{z}=0 \quad$ and $\quad M(v) \rho_{0}=m_{0}$.

$\epsilon^{1}$ terms:

$$
\begin{aligned}
\nabla_{x} \cdot\left(v m_{0}\right) & +\alpha \operatorname{div}_{v}\left(\mathbb{B}(v)\left(\nabla_{x} q(\hat{v})-\nabla_{x} h\right) m_{0}\right)=\lambda_{0}\left(M(v) \rho_{1}-m_{1}\right)+\lambda_{1}\left(M(v) \rho_{1}^{z}-m_{1}^{z}\right) \\
\nabla_{x} \cdot\left(v m_{0}^{z}\right) & +\alpha \operatorname{div}_{v}\left(\mathbb{B}(v)\left(\nabla_{x} q(\hat{v})-\nabla_{x} h\right) m_{0}^{z}\right) \\
& +\left(k_{+} Q+k_{-}\right) m_{1}^{z}+f^{\prime}(Q) v \cdot \nabla Q m_{0}=\lambda_{0}\left(M(v) \rho_{1}^{z}-m_{1}^{z}\right) .
\end{aligned}
$$

Integrating with respect to $v$ we obtain (for undirected tissue)

$$
\begin{aligned}
\rho_{1}^{z} & =0, \quad m_{1}^{z}=-\frac{M(v) \rho_{0}}{k_{+} Q+k_{-}+\lambda_{0}} f^{\prime}(Q) v \cdot \nabla Q \\
m_{1} & =\frac{1}{\lambda_{0}}\left[-\nabla_{x} \cdot\left(v M(v) \rho_{0}\right)-\alpha \operatorname{div}_{v}\left(\mathbb{B}(v)\left(\nabla_{x} q(t, x, \hat{v})-\nabla_{x} h\right) M(v) \rho_{0}\right)-\lambda_{1} m_{1}^{z}\right] \\
& +M(v) \rho_{1} .
\end{aligned}
$$

$\epsilon^{2}$ terms from (3.18a), after expanding $\mu$ about $\rho_{0}$ and integrating the equation with respect to $v$ :

$$
\partial_{t} \rho_{0}+\nabla_{x} \cdot \int_{V} v m_{1} d v=\mu\left(\rho_{0}, h\right) Q \rho_{0}
$$

From 3.20 we can compute

$$
\begin{aligned}
\int_{V} v m_{1} d v & =\frac{1}{\lambda_{0}}\left[-\nabla_{x} \cdot\left(\int_{V} v \otimes v M(v) d v \rho_{0}\right)\right. \\
& -\alpha \int_{V} v \operatorname{div}_{v}\left(\mathbb{B}(v)\left(\nabla_{x} q(t, x, \hat{v})-\nabla_{x} h(t, x)\right) M(v) \rho_{0}\right) d v \\
& \left.+\frac{\lambda_{1} f^{\prime}(Q)}{k_{+} Q+k_{-}+\lambda_{0}} \int_{V} v \otimes v M(v) d v \nabla Q \rho_{0}\right] .
\end{aligned}
$$


We denote by

$$
\tilde{\mathbb{E}}_{q}(t, x):=\int_{V} v \frac{q(t, x, \hat{v})}{\omega} \mathrm{d} v=s \int_{\mathbb{S}^{N-1}} \theta q(t, x, \theta) d \theta=s \mathbb{E}_{q}(t, x)
$$

and with

$$
\mathbb{D}_{T}(t, x):=\int_{V}\left(v-\tilde{\mathbb{E}}_{q}\right) \otimes\left(v-\tilde{\mathbb{E}}_{q}\right) M(t, x, v) \mathrm{d} v=\int_{V}\left(v-\tilde{\mathbb{E}}_{q}\right) \otimes\left(v-\tilde{\mathbb{E}}_{q}\right) \frac{q(t, x, \hat{v})}{\omega} \mathrm{d} v
$$

the so-called tumor diffusion tensor ${ }^{5}$. In the situation with undirected tissue we have $\mathbb{E}_{q}(t, x)=0$, thus in this case $\mathbb{D}_{T}(t, x)=s^{2} \mathbb{V}_{q}(t, x)$, where $\mathbb{V}_{q}$ denotes the variance-covariance matrix w.r.t. the fiber orientation distribution $q$. With the notation

$$
g\left(Q, \lambda_{0}\right)(t, x):=\frac{f^{\prime}(Q)}{k_{+} Q+k_{-}+\lambda_{0}},
$$

we have

$$
\int_{V} v m_{1} d v=-\frac{1}{\lambda_{0}} \nabla_{x} \cdot\left(\mathbb{D}_{T} \rho_{0}\right)+\frac{\lambda_{1}}{\lambda_{0}} g\left(Q, \lambda_{0}\right) \mathbb{D}_{T} \nabla Q \rho_{0}+\frac{\alpha}{\lambda_{0}} \Sigma(t, x) \rho_{0},
$$

with $\Sigma(t, x):=S_{1}(t, x ; q)-S_{2}(t, x ; h)$, where

$$
\begin{aligned}
& S_{1}(t, x ; q):=\int_{V} \mathbb{B}(v) \nabla_{x} q(t, x, \hat{v}) M(t, x, v) d v=\frac{1}{\omega} \int_{V} \mathbb{B}(v) \nabla_{x} q(t, x, \hat{v}) q(t, x, \hat{v}) d v \\
& S_{2}(t, x ; h):=\int_{V} \mathbb{B}(v) M(t, x, v) d v \nabla_{x} h(t, x)=s^{2}\left(\mathbb{I}_{N}-\mathbb{V}_{q}(t, x)\right) \nabla_{x} h(t, x) .
\end{aligned}
$$

Then, the limiting macroscopic equation for the tumor cell density takes the form

$$
\begin{aligned}
\partial_{t} \rho_{0}= & \nabla \cdot\left[\frac { 1 } { \lambda _ { 0 } ( x ) } \left(\nabla \cdot\left(\mathbb{D}_{T} \rho_{0}\right)-\lambda_{1}(x) g\left(Q, \lambda_{0}\right) \mathbb{D}_{T} \nabla Q \rho_{0}-\alpha S_{1}(t, x ; q) \rho_{0}\right.\right. \\
& \left.\left.+\alpha S_{2}(t, x ; h) \rho_{0}\right)\right]+\mu\left(\rho_{0}, h\right) Q \rho_{0} .
\end{aligned}
$$

The obtained equation is of drift-diffusion type; the first term on the right hand side represents a non-Fickian, so-called myopic diffusion: the cells spread out according to information available in their immediate surroundings. The next two terms feature a sign opposite to the myopic diffusion and represent drift corrections of the diffusive part in the direction of the macroscopic and mesoscopic tissue gradients $\nabla Q$ and $\nabla q$, respectively. They could therefore be interpreted as haptotaxis-like terms. The first of them carries through the function $g$ the influence of the subcellular receptor binding dynamics to the surrounding tissue, while the remaining drift term accounts for the (mesoscopic) stress exerted by the cells on the tissue, the latter being described by the directional distribution of fibers $q$. The drift term involving $S_{2}$ in 3.25 has the same sign with the diffusion, and contains a bias towards the opposite direction of acidity gradient $\nabla h$, thus describes a repellent $\mathrm{pH}$-taxis. The information about the brain tissue structure, which is decisive for personalized predictions of the tumor space-time evolution, is contained in (3.25) via the tumor 'diffusion' tensor $\mathbb{D}_{T}$ and the vector $S_{1}(t, x ; q)$, which in view of $(2.4)$ can be seen as a biomechanical interaction force between tumor cells and tissue. Thereby, the stress tensor $\mathbb{B}(v)$ contributes (together with the kernel $M(t, x, v)=\frac{q(t, x, \hat{v})}{\omega}$ ) to the (mesoscopic) haptotactic sensitivity of the cells, which is expressed here in a velocity-averaged way. The last term in (3.25 encodes proliferation and decay of glioma embedded in tissue. The function $\mu\left(\rho_{0}, h\right)$ can be correspondingly chosen, e.g. in the form

$$
\mu\left(\rho_{0}, h\right)=\eta\left(1-\rho_{0}-n\right)\left(h_{T}-h\right)_{+}-\gamma h,
$$

which would correspond to a logistic type of growth and a hypoxia-triggered necrosis. Thereby $\eta, \gamma>0$ are constants and $h_{T}$ denotes an acidity threshold which is critical for the cancer cells:

\footnotetext{
${ }^{5}$ This designation is somewhat abusive, since here -unlike previous works 252772 - the turning rate is not involved in its expression, due to its dependence on the position. When writing out the myopic diffusion it becomes evident that the 'actual' diffusion tensor is $\mathbb{D}_{T} / \lambda_{0}$.
} 
when the proton concentration exceeds it, they become hypoxic and cease proliferation. The choice of $\mu$ establishes the form of the last source term in 2.15 . Thus, with $(3.26)$ we have in the equation for necrotic tissue

$$
\mathcal{F}(h, \rho, Q)=\gamma h \rho Q
$$

The role of the turning rate $\lambda$ is twofold: it connects the cell reorientations to the receptor binding kinetics and it also captures the effect of tissue anisotropy on the cells migrating through the tissue. While $\lambda_{0}$ influences all motility terms, $\lambda_{1}$ is specific for the haptotaxis component including subcellular level effects. The factor $\frac{1}{\lambda_{0}}$ is independent on $y$ and increasing with the space-varying fractional anisotropy $F A$, the effect of which is particularly accentuated in the term multiplied with $\lambda_{1}$. This means that $\lambda_{1}$ with the inherent $y$-dependence provides an anisotropy-triggered switch between myopic diffusion with 'mesolevel' haptotaxis and migratory mode with enhanced haptotaxis, the latter supplementary providing bias in the direction of the tissue gradient. Observe that an almost isotropic tissue lets $\lambda_{0}$ be near constantly $\kappa$ and nearly turns off $\lambda_{1}$ and therewith the influence of subcellular dynamics. In this case, without the effect of $S_{1}(t, x ; q)$ there would only be myopic diffusion, as e.g., in 72 .

\subsubsection{Boundary conditions and the full macro-meso system}

So far we considered the space variable $x \in \mathbb{R}^{N}$, however the brain occupies a well delimited region inside the skull. Therefore we consider a bounded space domain $\Omega \subset \mathbb{R}^{N}$ and assume it to have a smooth enough boundary. Through the rescaling $x \rightarrow \varepsilon x$ the domain on which (3.25) holds is $\tilde{\Omega}=\varepsilon \Omega$, having outer unit normal vector $\nu(x)$ at $x \in \partial \tilde{\Omega}$. In order to determine the corresponding boundary conditions on $\partial \tilde{\Omega}$ we assume that there is no normal mass flux across the boundary [59, which translates into the mesoscopic no-flux condition 74

$$
\int_{V} v p(t, x, v, z) \cdot \nu(x) d v=0, \quad \text { for all } x \in \partial \tilde{\Omega}, t>0 .
$$

This condition actually means that the normal component of the macroscopic ensemble velocity $U(t, x)=\int_{V} v m(t, x, v) d v$ of the tumor across the space boundary vanishes (the tumor cannot leave the brain). Following [74] we write the boundary of the phase space as

$$
\partial \tilde{\Omega} \times V \times Z=\left(\Gamma_{+} \cup \Gamma_{-} \cup \Gamma_{0}\right) \times Z
$$

where

$$
\Gamma_{ \pm}:=\{(x, v) \in \partial \tilde{\Omega} \times V: \pm v \cdot \nu(x)>0\}, \quad \Gamma_{0}:=\{(x, v) \in \partial \tilde{\Omega} \times V: v \cdot \nu(x)=0\} .
$$

We assume that $\Gamma_{0}$ has zero measure w.r.t. the Lebesgue measure on $\partial \tilde{\Omega} \times V$ and consider the trace spaces

$$
L_{ \pm}^{2}:=L^{2}\left(\Gamma_{ \pm} \times Z ;|v \cdot \nu(x)| d \sigma(x) d v d z\right) .
$$

Moreover, $p$ is supposed to be regular enough so that we can define the traces $\left.p\right|_{\Gamma_{ \pm} \times Z} \in L_{ \pm}^{2}$, and that for a fixed $t>0$

$$
\left.p\right|_{\partial \tilde{\Omega} \times V \times Z}(t, x, v, z)=\lim _{\substack{\tilde{x} \in \tilde{\Omega} \\ \tilde{x} \rightarrow x}} p(t, \tilde{x}, z), \quad \text { for each } x \in \partial \tilde{\Omega} .
$$

Assuming that a regular Hilbert expansion is valid in $\tilde{\Omega}$ we can therefore compute the trace by simply passing to the corresponding limit in the Hilbert expansions for $p(t, x, v, z)$ and accordingly also for the moments 3.19 . Thus, the no-flux condition 3.28 becomes

$$
\int_{V} v\left(p_{0}(t, x, v, z)+\varepsilon p_{1}(t, x, v, z)\right) d v \cdot \nu(x)+O\left(\varepsilon^{2}\right)=0, \quad x \in \partial \tilde{\Omega}, z \in Z, t>0 .
$$

This condition should not depend on $\varepsilon$, therefore we should have

$$
\int_{V} v p_{j}(t, x, v) \cdot \nu(x) d v=0 \quad \text { for all } j \geq 0, x \in \partial \tilde{\Omega}, z \in Z, t>0 .
$$


Indeed, for $j=0$ we already have this condition satisfied for undirected tissue:

$$
\begin{aligned}
\int_{Z} \int_{V} v p_{0}(t, x, v, z) d v d z \cdot \nu(x) & =\int_{V} v m_{0}(t, x, v) d v \cdot \nu(x) \\
& =\int_{V} v \frac{q(t, x, \hat{v})}{\omega} \rho_{0}(t, x) d v \cdot \nu(x) \\
& =\rho_{0} \tilde{\mathbb{E}}_{q}(t, x) \cdot \nu(x)=0 .
\end{aligned}
$$

Next we look at the condition for $j=1$, more precisely at its integration w.r.t. $z$ :

$$
\begin{aligned}
\int_{Z} \int_{V} v p_{1}(t, x, v, z) d v d z \cdot \nu(x) & =\int_{V} v m_{1}(t, x, v) d v \cdot \nu(x) \\
& \stackrel{\sqrt[3.23]{-}}{=}\left(-\frac{1}{\lambda_{0}} \nabla_{x} \cdot\left(\mathbb{D}_{T} \rho_{0}\right)+\frac{\lambda_{1}}{\lambda_{0}} g\left(Q, \lambda_{0}\right) \mathbb{D}_{T} \nabla Q \rho_{0}+\frac{\alpha}{\lambda_{0}} \Sigma(t, x) \rho_{0}\right) \cdot \nu(x),
\end{aligned}
$$

which leads to a typical no-flux boundary condition

$$
\left(-\frac{1}{\lambda_{0}} \nabla_{x} \cdot\left(\mathbb{D}_{T} \rho_{0}\right)+\frac{\lambda_{1}}{\lambda_{0}} g\left(Q, \lambda_{0}\right) \mathbb{D}_{T} \nabla Q \rho_{0}+\frac{\alpha}{\lambda_{0}} \Sigma(t, x) \rho_{0}\right) \cdot \nu(x)=0 \quad \text { on } \partial \tilde{\Omega}, t>0
$$

for the macroscopic PDE (3.25). This equation is coupled with the dynamics of acidity $h$ by way of $S_{2}(t, x ; h)$ in $\Sigma(t, x)$. For this ab initio macroscopic equation we can directly impose a no-flux condition:

$$
D_{H} \nabla_{x} h=0 \quad \text { on } \partial \tilde{\Omega}, t>0 .
$$

In previous works $25,27,38,40]$ the quantities relating to the tissue, i.e. $q$ and (where applicable) $Q$ were assumed to be time-invariant, whereby $Q$ itself was estimated from the DTI data, as in 25 27, 42. No tissue degradation was accounted for, and one could also include a proliferation term, e.g. like in 26. In this much simplified situation only coupling (3.25) with 2.5 and using the no-flux boundary conditions given above, the system takes the form of a Keller-Segel problem with some supplementary drift terms, all of which are linear in $\rho_{0}$.

When the full evolution of the tissue becomes relevant, then 3.25 and 2.5 have to be supplementally coupled with the dynamics of $q$, as given by (2.14), and with that of $Q$, given by 2.11). The resulting (reaction-)diffusion-taxis system then characterizes cell, acidity, and tissue dynamics evolving on two scales (macroscopic and mesoscopic, respectively). Thereby, we deal with the macroscopic cell density in the first PDE, while 2.14) and 2.11) involve the mesoscopic quantity $p(t, x, v, y)$, which is inconvenient both for the analysis and the numerics.

Remark that, thanks to property 3 of $L_{0}$, the Hilbert expansion is equivalent to splitting $p$ as $p(t, x, v, y)=\bar{p}(t, x, y) M(t, x, v)+\varepsilon M^{\perp}(t, x, v)$, with $\int_{V} M^{\perp}(t, x, v) d v=0$, i.e. the ChapmanEnskog expansion used in [38]. The leading order of the operator in 2.8) leads to

$$
\Pi_{a}[q](t, x, \theta) \simeq \begin{cases}\int_{\mathbb{S}^{N-1}}\left|\theta \cdot \theta^{\prime}\right| q\left(t, x, \theta^{\prime}\right) d \theta^{\prime} & \text { for undirected tissue } \\ \int_{\mathbb{S}^{N-1}} \theta \cdot \theta^{\prime} q\left(t, x, \theta^{\prime}\right) d \theta^{\prime} & \text { for directed tissue }\end{cases}
$$

along with correspondingly rewriting 2.12 as

$$
A[q](t, x)=\int_{\mathbb{S}^{N-1}} \Pi_{a}[q](t, x, \theta) q(t, x, \theta) d \theta .
$$

Therewith we obtain from (2.14) the equation

$$
\partial_{t} q(t, x, \theta)=r_{D}(h) \rho(t, x) q(t, x, \theta)\left(\Pi_{a}[q](t, x, \theta)-A[q](t, x)\right)
$$

and from 2.13

$$
\partial_{t} Q=r_{D}(h) \rho Q(A[q](t, x)-1) .
$$

Thus, in order to determine the dynamics of tumor cells in interaction with the tissue they 
degrade and with the acidity they produce, we have to solve the macro-meso system

$$
\begin{aligned}
& \partial_{t} \rho=\nabla_{x} \cdot\left[\frac{1}{\lambda_{0}(t, x)}\left(\nabla_{x} \cdot\left(\mathbb{D}_{T} \rho\right)-\lambda_{1}(t, x) g\left(Q, \lambda_{0}\right) \mathbb{D}_{T} \nabla Q \rho-\alpha S_{1}(t, x ; q) \rho+\alpha S_{2}(t, x ; h) \rho\right)\right] \\
& \quad+\mu(\rho, h) Q \rho \\
& \partial_{t} q(t, x, \theta)=r_{D}(h) \rho(t, x) q(t, x, \theta)\left(\Pi_{a}[q](t, x, \theta)-A[q](t, x)\right) \\
& \partial_{t} Q=r_{D}(h) \rho Q(A[q]-1) \\
& h_{t}=D_{H} \Delta h+\frac{a \rho}{1+\rho}-b Q\left(h-h_{0}\right) \\
& \partial_{t} n=r_{D}(h) \rho Q(1-A[q])+\mathcal{F}(h, \rho, Q),
\end{aligned}
$$

where $\mathbb{D}_{T}$ is as in 3.22 with $\mathbb{E}_{q}=0$, with $S_{1}$ and $S_{2}$ as in $3.24, \Pi_{a}[q]$ as in (3.31), $A[q]$ as in (3.32), $\mu$ as in (3.26), $\mathcal{F}$ as in (3.27), with boundary conditions $(3.29),(3.30)$, and with given initial conditions $\rho(0, x), q(0, x, \theta), Q(0, x), h(0, x)$, and $n(0, x)$. These can be the tumor cell distribution (or an approximation of it) observed at diagnosis, the directional distribution of fibers obtained via DTI, some estimate of the macroscopic volume fraction of the tissue (e.g., most simply $F A$, as in 23,25$]$ ), some (estimated) acidity distribution at diagnosis, and the necrotic tissue distribution, respectively. A tumor segmentation of the diagnosis image could be useful in assessing the latter.

The mathematical handling of system (3.35) is nontrivial, both with respect to well posedness and numerics. The equations connect two modeling levels (macroscopic and mesoscopic) and the couplings via $q$ involved in the coefficients of all terms on the right hand side of (3.35a) render the problem highly nonlinear. Moreover, the equation for $\rho$ features (along with the myopic diffusion) three types of taxis:

- macroscopic haptotaxis towards $\nabla Q$,

- a new kind of mesoscopic haptotaxis (term with $S_{1}$ ), where the bias is actually given by $\nabla_{x} q^{2}$, and

- pH-taxis, describing chemorepellence due to acidity (term with $S_{2}$ ).

\subsection{Moment closure for the fiber equation}

The system (3.35) is macroscopic with respect to the cell dynamics: by an asymptotic expansion of $p(t, x, v, y)$ around the local Maxwellian $q(t, x, \theta)$, we derived a model for the local cell density $\rho(t, x)$. However, the tissue is modeled by the mesoscopic quantity $q(t, x, \theta)$. To reduce the level of detail in the tissue dynamics to match the rest of the model, we derive a low-order moment approximation to $3.35 \mathrm{~b}$. For simplicity, we only consider the two-dimensional case.

Recall the peanut distribution 38,72 :

$$
q(\theta)=\frac{1}{\left\langle\theta^{\top} \mathbb{D}_{W} \theta\right\rangle} \theta^{\top} \mathbb{D}_{W} \theta=\frac{1}{\pi \operatorname{tr} \mathbb{D}_{W}} \theta^{\top} \mathbb{D}_{W} \theta .
$$

We interpret this as a $P_{2}$-approximation 15$]$ [5, III.5] under some additional constraints. A second-order basis of monomials is given by

$$
\boldsymbol{a}=\left(\begin{array}{lllll}
\theta_{x} & \theta_{y} & \theta_{x}^{2} & \theta_{x} \theta_{y} & \theta_{y}^{2}
\end{array}\right) .
$$

We drop the constant function from the basis, because it would introduce a linear dependence: $\theta_{x}^{2}+\theta_{y}^{2}=1$. The $P_{2}$ ansatz is defined by the linear combination of basis functions

$$
\mathfrak{q}(\theta)=\boldsymbol{\alpha} \cdot \boldsymbol{a},
$$

wherein $\boldsymbol{\alpha}$ is a vector of multipliers such that the moment constraints

$$
\boldsymbol{u}:=\langle\boldsymbol{a} q\rangle=\langle\boldsymbol{a} \mathfrak{q}\rangle=\left\langle\boldsymbol{a} \boldsymbol{a}^{\top}\right\rangle \mathfrak{k}
$$

are fulfilled. Due to the symmetry constraints $\langle\theta q\rangle=0$, we drop the first-order monomials $\theta_{x}, \theta_{y}$ from the basis. The remaining basis functions are then:

$$
\boldsymbol{b}=\left(\begin{array}{lll}
\theta_{x}^{2} & \theta_{x} \theta_{y} & \theta_{y}^{2}
\end{array}\right) .
$$


With this choice it is easy to associate the multipliers $\beta$ in the ansatz

$$
\mathfrak{q}=\beta \cdot \boldsymbol{b}(\theta)
$$

with the components of the normalized water diffusion tensor:

$$
\beta:=\left(\begin{array}{lll}
\beta_{x x} & \beta_{x y} & \beta_{y y}
\end{array}\right)=\frac{1}{\pi \operatorname{tr} \mathbb{D}_{W}}\left(\begin{array}{lll}
\mathbb{D}_{W x x} & 2 \mathbb{D}_{W x y} & \mathbb{D}_{W y y}
\end{array}\right) .
$$

Moreover, the moments $\boldsymbol{w}:=\langle\boldsymbol{b} q\rangle$ are directly related to the components of the (normalized) tumor diffusion tensor $P=\left\langle\theta \theta^{\top} q\right\rangle$ :

$$
\boldsymbol{w}:=\left(\begin{array}{lll}
w_{x x} & w_{x y} & w_{y y}
\end{array}\right)=\left(\begin{array}{lll}
P_{x x} & P_{x y} & P_{y y}
\end{array}\right) .
$$

We can translate multipliers to moments with the moment constraints $\boldsymbol{w}=\langle\boldsymbol{b} \mathfrak{q}\rangle=\left\langle\boldsymbol{b} \boldsymbol{b}^{\top}\right\rangle \boldsymbol{\beta}$. The transfer matrix is given by

$$
H:=\left\langle\boldsymbol{b}^{\top}\right\rangle=\frac{\pi}{4}\left(\begin{array}{ccc}
3 & 0 & 1 \\
0 & 1 & 0 \\
1 & 0 & 3
\end{array}\right) .
$$

With these tools in hand, we derive a $P_{2}$-approximation of the tissue dynamics $\left.3.35 \mathrm{~b}\right)$ through the following steps: Insert the ansatz $\mathfrak{q}$ into $(3.35 \mathrm{~b}$ ), multiply by the basis $\boldsymbol{b}$ and integrate over $\mathbb{S}^{1}$ to obtain

$$
\partial_{t} \boldsymbol{w}=r_{D}(h) \rho(\langle\boldsymbol{b} \mathfrak{q} \Pi[\mathfrak{q}]\rangle-\boldsymbol{w} A[\mathfrak{q}]) .
$$

It remains to calculate the moments $\langle\boldsymbol{b} \mathfrak{q} \Pi[\mathfrak{q}]\rangle$. Inserting the definitions of $\mathfrak{q}$ and $\Pi$, we obtain

$$
\langle\boldsymbol{b} \mathfrak{q} \Pi[\mathfrak{q}]\rangle=\int_{\mathbb{S}^{1}} \int_{\mathbb{S}^{1}} \boldsymbol{b}(\theta) \boldsymbol{\beta} \cdot \boldsymbol{b}(\theta) \boldsymbol{\beta} \cdot \boldsymbol{b}\left(\theta^{\prime}\right)\left|\theta \cdot \theta^{\prime}\right| \mathrm{d} \theta^{\prime} \mathrm{d} \theta .
$$

With the tensor

$$
\mathfrak{P}_{k, i j}=\int_{\mathbb{S}^{1}} \int_{\mathbb{S}^{1}} b_{k}(\theta) b_{i}(\theta) b_{j}\left(\theta^{\prime}\right)\left|\theta \cdot \theta^{\prime}\right| \mathrm{d} \theta^{\prime} \mathrm{d} \theta
$$

we can write the $k$-th component $\langle\boldsymbol{b} \mathfrak{q} \Pi[\mathfrak{q}]\rangle_{k}$ as

$$
\langle\boldsymbol{b} \mathfrak{q} \Pi[\mathfrak{q}]\rangle_{k}=\boldsymbol{\beta}^{\top} \mathfrak{P}_{k} \boldsymbol{\beta}=\boldsymbol{w}^{\top}\left(H^{-1 \top} \mathfrak{P}_{k} H^{-1}\right) \boldsymbol{w} .
$$

Finally, we obtain $A[\mathfrak{q}]$ from the identity $\theta_{x}^{2}+\theta_{y}^{2}=1$ :

$$
A[\mathfrak{q}]=\langle 1 \mathfrak{q} \Pi[\mathfrak{q}]\rangle=\left\langle\left(\theta_{x}^{2}+\theta_{y}^{2}\right) \mathfrak{q} \Pi[\mathfrak{q}]\right\rangle=\langle\boldsymbol{b} \mathfrak{q} \Pi[\mathfrak{q}]\rangle_{x x}+\langle\boldsymbol{b} \mathfrak{q} \Pi[\mathfrak{q}]\rangle_{y y} .
$$

The tensor $\mathfrak{P}_{k, i j}$ can be precomputed once and for all with a high-order quadrature. To evaluate each component of the right-hand side of (3.36) we need to compute only a quadratic form at run time. Note that the normalization $\langle q\rangle=1$ results in the loss of an additional degree of freedom. It holds tr $P=1$, therefore we can reconstruct $w_{y y}=1-w_{x x}$ and only need the evolve the two moments $w_{x x}, w_{x y}$ in 3.36 .

Remark: In three dimensions, the previous considerations are completely analogous for the basis $\boldsymbol{b}=\left(\begin{array}{llllll}\theta_{x}^{2} & \theta_{y}^{2} & \theta_{z}^{2} & \theta_{x} \theta_{y} & \theta_{x} \theta_{z} & \theta_{y} \theta_{z}\end{array}\right)$.

\subsection{Hyperbolic scaling, directed tissue}

Here we also aim to investigate the effect of reducing diffusivity that might not be experimentally consistent. In order to achieve this objective and for the sake of completeness, we propose in this subsection to perform a hyperbolic limit that will provide us with a macroscopic vision in which the terms of transport and potentials win the battle over diffusion. We will take advantage of most of the calculations in Subsection 3.1, that we will omit in part so as not to be repetitive. Finally, in order to try to model a series of effects of a priori minor influence according to the experiments, not contemplated in the model variables, we carry out a "small" extension to the 
second order of the hyperbolic expansion and compare this double development on the scale with the parabolic approach.

In the following we address a version of (3.16) where we neglect the dependence of the turning rate on the subcellular dynamics, i.e. we consider it to be of the form $\lambda(x)=\frac{\kappa}{F A(x)+1}$ and are interested in the situation of directed tissue.

We thus consider the kinetic transport equation

$$
\begin{aligned}
\partial_{t} p & +v \cdot \nabla_{x} p-\partial_{z}\left(\left(\left(k_{+} Q+k_{-}\right) z+f^{\prime}(Q)\left(Q_{t}+v \cdot \nabla Q\right)\right) p\right)+\alpha \operatorname{div}_{v}\left(\mathbb{B}(v)\left(\nabla_{x} q(\hat{v})-\nabla_{x} h\right) p\right) \\
& =\lambda(x) L_{0}(p)+\mathcal{P}(p, Q, h, \rho),
\end{aligned}
$$

with $L_{0}(p)=\bar{p}(t, x, z) \frac{q(x, \hat{v})}{\omega}-p(t, x, v, z)$ having the same properties as in Subsection 3.1 and perform a hyperbolic scaling $t \rightarrow \varepsilon t, x \rightarrow \varepsilon x$, while the proliferation term is rescaled as previously with $\varepsilon^{2}$. This leads to

$$
\begin{aligned}
\varepsilon \partial_{t} p & +\varepsilon v \cdot \nabla_{x} p-\partial_{z}\left(\left(\left(k_{+} Q+k_{-}\right) z+\varepsilon f^{\prime}(Q)\left(Q_{t}+v \cdot \nabla Q\right)\right) p\right)+\alpha \varepsilon \operatorname{div}_{v}\left(\mathbb{B}(v)\left(\nabla_{x} q(\hat{v})-\nabla_{x} h\right) p\right) \\
& =\lambda(x) L_{0}(p)+\varepsilon^{2} \mathcal{P}(p, Q, h, \rho) .
\end{aligned}
$$

We now consider a Chapman-Enskog expansion (equivalent here to the Hilbert one, as stated in Subsection 3.1p, i.e., a decomposition of $p$ into a $\operatorname{Ker}\left(L_{0}\right)$-component and a $\operatorname{Ker}\left(L_{0}\right)^{\perp}$-part, as follows:

$$
p(t, x, v, z)=\bar{p}(t, x, z) \frac{q(t, x, \hat{v})}{\omega}+\varepsilon p^{\perp}(t, x, v, z),
$$

where $p^{\perp} \in\left\langle\frac{q}{\omega}\right\rangle^{\perp}$ verifies $\int_{V} p^{\perp}(t, x, v, z) d v=0$. This decomposition leads to the corresponding expansion for the moments of $p$, in particular: $m(t, x, v)=\rho(t, x) \frac{q(t, x, \hat{v})}{\omega}+\varepsilon m^{\perp}(t, x, v, z)$, and then, integrating $(3.38)$ w.r.t. $z \in Z$ we obtain

$$
\begin{array}{r}
\varepsilon \partial_{t} \rho \frac{q}{\omega}+\varepsilon \rho \partial_{t}\left(\frac{q}{\omega}\right)+\varepsilon^{2} \partial_{t} m^{\perp}+\varepsilon v \cdot \nabla_{x}\left(\rho \frac{q}{\omega}\right)+\varepsilon^{2} v \cdot \nabla_{x} m^{\perp}+\alpha \varepsilon \nabla_{v} \cdot\left(\mathbb{B}(v)\left(\nabla_{x} q(\hat{v})-\nabla_{x} h\right) m\right) \\
=\varepsilon \lambda(x) L_{0}\left(m^{\perp}\right)+\varepsilon^{2} \mu(\rho, h) Q m .
\end{array}
$$

Integrate w.r.t. $v$ and divide by $\varepsilon$ to get

$$
\rho_{t}+\nabla_{x} \cdot\left(\rho \tilde{\mathbb{E}}_{q}+\varepsilon \int_{V} v m^{\perp} d v\right)=\varepsilon \rho \mu(\rho, h) Q,
$$

where as before $\tilde{\mathbb{E}}_{q}(t, x)=\int_{V} v \frac{q(t, x, \hat{v})}{\omega} d v=s \mathbb{E}_{q}(t, x)$.

Clearly 3.41 is drift-dominated; however, it is worth computing the $\mathcal{O}(\varepsilon)$ correction with respect to the pure drift. From 3.40 and 3.41 follows

$$
\begin{aligned}
\lambda(x) L_{0}\left(m^{\perp}\right) & =\varepsilon \frac{q}{\omega} \rho \mu(\rho, h) Q-\frac{q}{\omega} \nabla_{x} \cdot\left(\rho \tilde{\mathbb{E}}_{q}+\varepsilon \int_{V} v m^{\perp} d v\right)+\rho \frac{\partial_{t} q}{\omega}+\varepsilon \partial_{t} m^{\perp}+v \cdot \nabla_{x}\left(\rho \frac{q}{\omega}+\varepsilon m^{\perp}\right) \\
+ & \alpha \nabla_{v} \cdot\left(\mathbb{B}(v)\left(\nabla_{x} q(\hat{v})-\nabla_{x} h\right) m\right)-\varepsilon \rho \mu(\rho, h) Q .
\end{aligned}
$$

Now observe that the integral w.r.t. $v$ of the right hand side in 3.42 vanishes, so that we can take the (pseudo)inverse of $L_{0}$ giving (at leading order)

$$
\begin{aligned}
m^{\perp} \simeq & -\frac{1}{\lambda(x)}\left[\frac{q}{\omega}\left(v-\tilde{\mathbb{E}}_{q}\right) \cdot \nabla \rho+\rho\left(v \cdot \nabla_{x} \frac{q}{\omega}-\frac{q}{\omega} \nabla_{x} \cdot \tilde{\mathbb{E}}_{q}\right)+\rho \frac{\partial_{t} q}{\omega}\right. \\
& \left.+\alpha \nabla_{v} \cdot\left(\mathbb{B}(v)\left(\nabla_{x} q(\hat{v})-\nabla_{x} h\right) m\right)\right],
\end{aligned}
$$

hence

$$
\begin{aligned}
\int_{V} v m^{\perp} d v & \simeq-\frac{1}{\lambda(x)}\left(\int_{V} v \otimes\left(v-\tilde{\mathbb{E}}_{q}\right) \frac{q}{\omega} d v \nabla \rho\right. \\
& \left.+\rho\left(\nabla_{x} \cdot \int_{V} v \otimes v \frac{q}{\omega} d v-\tilde{\mathbb{E}}_{q} \nabla_{x} \cdot \tilde{\mathbb{E}}_{q}\right)+\rho \partial_{t} \tilde{\mathbb{E}}_{q}-\alpha \rho \Sigma(t, x)\right) \\
& =-\frac{1}{\lambda(x)}\left(\nabla_{x} \cdot\left(\mathbb{D}_{T} \rho\right)-\rho \tilde{\mathbb{E}}_{q} \nabla_{x} \cdot \tilde{\mathbb{E}}_{q}+\rho \partial_{t} \tilde{\mathbb{E}}_{q}-\alpha \rho \Sigma(t, x)\right)
\end{aligned}
$$


where $\mathbb{D}_{T}(t, x)$ and $\Sigma(t, x):=S_{1}(t, x ; q)-S_{2}(t, x ; h)$ are as introduced in Section 3.1 and we observe that

$$
\int_{V} v \otimes\left(v-\tilde{\mathbb{E}}_{q}\right) \frac{q}{\omega} d v=\int_{V}\left(v-\tilde{\mathbb{E}}_{q}\right) \otimes\left(v-\tilde{\mathbb{E}}_{q}\right) \frac{q}{\omega} d v=\mathbb{D}_{T}
$$

Together with (3.41) this leads to the macroscopic PDE

$$
\rho_{t}+\nabla \cdot\left(\rho \tilde{\mathbb{E}}_{q}\right)=\varepsilon \nabla \cdot\left(\frac{1}{\lambda(x)}\left(\nabla \cdot\left(\rho \mathbb{D}_{T}(t, x)\right)+\rho\left(\partial_{t} \tilde{\mathbb{E}}_{q}-\tilde{\mathbb{E}}_{q} \nabla \cdot \tilde{\mathbb{E}}_{q}-\alpha \Sigma(t, x)\right)\right)\right)+\varepsilon \rho \mu(\rho, h) Q,
$$

which is drift-dominated. Notice that for $\mathbb{E}_{q}=0$, i.e. if the tissue is undirected, the correction term in (3.47) has the same form as the right hand side of (3.25) - except for the middle term therein, which got lost when integrating (3.38) w.r.t. $z$. In fact, it was the assumption of the turning rate not depending on $z$ which effaced the whole influence of subcellular dynamics. The effect of this is not having the macroscopic haptotaxis term in the $\varepsilon$-correction on the right hand side. Nevertheless we still get the pH-taxis and (mesoscopic) haptotaxis correction terms contained in

$$
\varepsilon \nabla \cdot\left(\frac{\alpha}{\lambda(x)} \rho \Sigma(t, x)\right)=\varepsilon \nabla \cdot\left(\frac{\alpha}{\lambda(x)} \rho\left(S_{1}(t, x ; q)-S_{2}(t, x ; h)\right)\right) .
$$

Coupling (3.47) with the equations (3.35b) and (3.35d) describing tissue and acidity evolution, respectively, leads to challenges similar to those in Section 3.1 .

We need to prescribe boundary conditions. To this aim we consider again a bounded, sufficiently smooth domain $\tilde{\Omega}=\varepsilon \Omega \subset \mathbb{R}^{N}$ and start from a mesoscopic no-flux condition of the form (3.28). Using the Chapman-Enskog expansion (3.39) of $p(t, x, v, z)$ we rewrite this condition as

$$
\left(\rho(t, x) \tilde{\mathbb{E}}_{q}(x)+\varepsilon \int_{Z} \int_{V} v p^{\perp}(t, x, v, z) d v d z\right) \cdot \nu(x)=0, \quad \text { for all } x \in \partial \tilde{\Omega}, t>0,
$$

which in virtue of 3.45 leads to

$$
\left(-\rho \tilde{\mathbb{E}}_{q}+\frac{\varepsilon}{\lambda(x)}\left(\nabla \cdot\left(\rho \mathbb{D}_{T}(t, x)\right)+\rho\left(\partial_{t} \tilde{\mathbb{E}}_{q}-\tilde{\mathbb{E}}_{q} \nabla \cdot \tilde{\mathbb{E}}_{q}-\alpha \Sigma(t, x)\right)\right)\right) \cdot \nu(x)=0, \quad x \in \partial \tilde{\Omega}, t>0,
$$

meaning that the normal macroscopic flux and its $\varepsilon$-correction vanish on the spatial boundary.

Thus, in the case with directed tissue and when no subcellular dynamics are taken into account, the macro-meso system to be solved becomes

$\rho_{t}+s \nabla \cdot\left(\rho \mathbb{E}_{q}\right)=\varepsilon \nabla \cdot\left(\frac{s^{2}}{\lambda(x)}\left(\nabla \cdot\left(\rho \mathbb{V}_{q}(t, x)\right)+\rho\left(\frac{1}{s} \partial_{t} \mathbb{E}_{q}-\mathbb{E}_{q} \nabla \cdot \mathbb{E}_{q}-\frac{\alpha}{s^{2}} \Sigma(t, x)\right)\right)\right)+\varepsilon \rho \mu(\rho, h) Q$

$h_{t}=D_{H} \Delta h+\frac{a \rho}{1+\rho}-b Q\left(h-h_{0}\right)$

$\partial_{t} q(t, x, \theta)=r_{D}(h) \rho(t, x) q(t, x, \theta)(\Pi[p](t, x, \theta)-A[p](t, x))$,

$\partial_{t} Q=r_{D}(h) \rho Q(A[p]-1)$

$\partial_{t} n=r_{D}(h) \rho Q(1-A[p])+\mathcal{F}(h, \rho, Q)$,

with $\Pi[p]$ and $A[p]$ as given in (2.8) and (2.12), respectively. Observe that upon assuming the tisue to be undirected (i.e. that $\mathbb{E}_{q}=0$ ), (3.49a) takes the form of (3.35a), which was obtained by parabolic scaling, with the difference of the right hand side of the glioma dynamics being scaled here by $\varepsilon$.

As for the parabolic limit, in (3.49) the dependence of the featured operators on the mesoscopic cell distribution function $p$ is inconvenient, so we use the same approach as in Subsection 3.1.1 to approximate $\Pi[p]$ and $A[p]$ by (3.31) and (3.32), respectively. Notice that in the present case (directed tissue) we can rewrite

$$
\begin{aligned}
\Pi_{a}[q](t, x, \theta) & =\theta \cdot \mathbb{E}_{q}(t, x) \\
A[q](t, x) & =\mathbb{E}_{q}(t, x) \cdot \mathbb{E}_{q}(t, x)
\end{aligned}
$$


Consequently, 3.49c can be replaced by

$$
\partial_{t} q(t, x, \theta)=r_{D}(h) \rho(t, x) q(t, x, \theta)\left(\theta \cdot \mathbb{E}_{q}(t, x)-\mathbb{E}_{q}^{2}(t, x)\right),
$$

hence we can compute

$$
\partial_{t} \mathbb{E}_{q}=r_{D}(h) \rho\left(\mathbb{V}_{q}+\mathbb{E}_{q} \otimes \mathbb{E}_{q}-\mathbb{E}_{q}^{2} \mathbb{I}_{N}\right) \cdot \mathbb{E}_{q}
$$

and plug it into 3.49a). Let us denote

$$
\mathbb{T}_{q}:=\frac{1}{s} r_{D}(h) \rho\left(\mathbb{V}_{q}+\mathbb{E}_{q} \otimes \mathbb{E}_{q}-\mathbb{E}_{q}^{2} \mathbb{I}_{N}\right) \cdot \mathbb{E}_{q}-\mathbb{E}_{q} \nabla \cdot \mathbb{E}_{q}
$$

Then altogether the system becomes

$$
\begin{aligned}
& \rho_{t}+\nabla \cdot\left(\rho \mathbb{E}_{q}\right)=\varepsilon \nabla \cdot\left(\frac{s^{2}}{\lambda(x)}\left(\nabla \cdot\left(\rho \mathbb{V}_{q}(t, x)\right)+\rho\left(\mathbb{T}_{q}-\frac{\alpha}{s^{2}} \Sigma(t, x)\right)\right)\right)+\varepsilon \rho \mu(\rho, h) Q \\
& h_{t}=D_{H} \Delta h+\frac{a \rho}{1+\rho}-b Q\left(h-h_{0}\right) \\
& \partial_{t} q=r_{D}(h) \rho q\left(\theta \cdot \mathbb{E}_{q}-\mathbb{E}_{q}^{2}\right) \\
& \partial_{t} Q=r_{D}(h) \rho Q\left(\mathbb{E}_{q}^{2}-1\right) \\
& \partial_{t} n=r_{D}(h) \rho Q\left(1-\mathbb{E}_{q}^{2}\right)+\mathcal{F}(h, \rho, Q)
\end{aligned}
$$

with $\theta \in \mathbb{S}^{N-1}, t>0$, and $x \in \tilde{\Omega}$. The functions $\mu(\rho, h)$ and $\mathcal{F}(h, \rho, Q)$ are still those given in (3.26) and (3.27), respectively. The equations are completed by initial conditions as in Subsection 3.1 .1 and by the no-flux boundary conditions $\nabla h \cdot \nu=0$ on $\partial \tilde{\Omega}$ and

$$
\left(-s \rho \mathbb{E}_{q}+\frac{s^{2} \varepsilon}{\lambda(x)}\left(\nabla \cdot\left(\rho \mathbb{V}_{q}(t, x)\right)+\rho\left(\mathbb{T}_{q}(t, x)-\frac{\alpha}{s^{2}} \Sigma(t, x)\right)\right)\right) \cdot \nu(x)=0, \quad x \in \partial \tilde{\Omega}, t>0 .
$$

\section{Numerics}

In this section the equations obtained by parabolic upscaling are solved numerically. Simulations are conducted for three scenarios of increasing complexity. In each scenario we describe a set of assumptions simplifying the equations. This allows for directly investigating the relative importance of the different effects included in our model. The results are presented in Subsection 4.3 . For convenience we state in brevity the exact macroscopic systems of PDEs for the considered scenarios:

Scenario 1: We assume that neither $q$ nor $Q$ depend on time, thus can be assessed from the DTI data. We follow previous works, starting from a pre-assigned directional distribution $q$ (peanut) and independently estimate $Q$ using the approach in $26,41,42$ relying on Brownian motion description of water molecule diffusion ${ }^{6}$ The system then reduces to (3.35a), (3.35d), merely featuring myopic diffusion, pH-taxis, and transport terms with drift velocities computed from the data, together with the necrosis dynamics $3.35 \mathrm{e}$ with $r_{D}(h)=0$, i.e.

$$
\begin{aligned}
\partial_{t} \rho= & \nabla_{x} \cdot\left[\frac{1}{\lambda_{0}(x)}\left(\nabla_{x} \cdot\left(\mathbb{D}_{T} \rho\right)-\lambda_{1}(x) g\left(Q, \lambda_{0}\right) \mathbb{D}_{T} \nabla Q \rho-\alpha S_{1}(t, x ; q) \rho+\frac{\alpha}{h_{0}} S_{2}(t, x ; h) \rho\right)\right] \\
& +Q \rho \eta(1-\rho-n) \frac{\left(h_{T}-h\right)_{+}}{h_{0}}-\gamma \frac{h}{h_{0}} \rho Q \\
\partial_{t} h= & D_{H} \Delta h+\frac{a \rho}{1+\rho}-b Q\left(h-h_{0}\right) \\
\partial_{t} n= & \mathcal{F}(h, \rho, Q) .
\end{aligned}
$$

\footnotetext{
${ }^{6}$ Alternative, more precise ways to assess the (macroscopic and/or mesoscopic) tissue density from DTI data would be to perform density estimation by using statistical or variational methods, see e.g. 24, 30, 50, 90. Here we do not address this issue, as our focus is on providing a continuous approach to modeling glioma growth and spread which is amenable to efficient computations and at the same time able to capture lower scale effects.
} 
Scenario 2: $Q$ evolves in time, meaning that the tissue is degrading irrespective of the fibre orientations (hence the anisotropy does not play any role in the degradation). Here $A[q]$ is zero. This corresponds to an indirect depletion, i.e. not upon contact with glioma cells, but rather through the acidity they produce. Consequently, the macroscopic system 3.35 simplifies to

$$
\begin{aligned}
\partial_{t} \rho= & \nabla_{x} \cdot\left[\frac{1}{\lambda_{0}(x)}\left(\nabla_{x} \cdot\left(\mathbb{D}_{T} \rho\right)-\lambda_{1}(x) g\left(Q, \lambda_{0}\right) \mathbb{D}_{T} \nabla Q \rho-\alpha S_{1}(t, x ; q) \rho+\alpha S_{2}(t, x ; h) \rho\right)\right] \\
& +Q \rho \eta(1-\rho-n) \frac{\left(h_{T}-h\right)_{+}}{h_{0}}-\gamma \frac{h}{h_{0}} \rho Q \\
\partial_{t} Q= & -r_{D}(h) \rho Q \\
\partial_{t} h= & D_{H} \Delta h+\frac{a \rho}{1+\rho}-b Q\left(h-h_{0}\right) \\
\partial_{t} n= & r_{D}(h) \rho Q+\mathcal{F}(h, \rho, Q) .
\end{aligned}
$$

Scenario 3: Both $q$ and $Q$ evolve and the full system 3.35 is solved.

\subsection{Numerical discretization}

The equations were discretized by the method of lines approach in all three cases. For the spatial discretization we used a vertex centered finite volume method. The mesh is naturally structured because of the underlying medical dataset. The mesh width $h=2 \mathrm{~mm}$ is imposed by the particular DTI dataset, which is publicly available 17]. The reconstructed diffusion tensors were averaged with a component-wise arithmetic mean at the control volume interfaces. The advective fluxes were approximated by a first order upwind discretization. The no-flux barrier between the brain and the background were implemented by suppressing flux assembly at the relevant cell interfaces? The integration of the reaction terms coupling the evolving fields was performed by a first order midpoint rule at the cell centers. As an initial condition we used a small Gaußian peak as an approximation of the tumor and took for the spatial distribution of the acidity field $h$ the uniform steady-state of (3.35d). The initial condition for necrotic matter was simply $n=0$. The time discretization was realized by an implicit Euler scheme. The resulting nonlinear system was solved via Newton iteration which internally used a BiCGSTAB solver preconditioned via AMG with an SSOR smoother [83].

\subsubsection{Software}

The implementation was performed within the DUNE software framework [3], specifically with the dune-pdelab discretization module 5:12. The BiCGSTAB solver and the AMG were provided by the iterative solver template library dune-istl 11 .

\subsection{Choice of simulation parameters and coefficient functions}

In Table 1 we present the parameters used throughout all numerical simulations, their meaning, and their respective sources. We can also assess the parabolic scaling parameter

$$
\varepsilon=\frac{s}{\kappa X} \simeq 2 \cdot 10^{-6},
$$

where we use $X=0.06 \mathrm{~m}$ as a reference length, corresponding to the side length of he plots in e.g., Figure 2p. This value indicates that the parabolic limit is an adequate approximation to the kinetic model for the chosen parameters.

Let us also highlight here the role of the scaling parameter $\alpha$. It is influenced by the position gradients of $q$ and $h$ (involved in $\Sigma$ ) and hence should be a large quantity, say of the order $\frac{1}{\gamma} \varepsilon^{-\gamma}$ for some $\gamma>0$. This is similar to what happens when we perform hyperbolic scaling in a fairly similar kinetic framework (see 8,77), where hydrodynamic limits with dominant drift are obtained, but where the small diffusion correction of a non-diffusive limit can be made explicit by displaying the dependency of $\varepsilon$ on all scaling parameters. In the present context $\gamma$ should be a parameter related to smaller scales, such as the microtubule (MT) extension zones that are responsible for the biochemical and biomechanical exchange of cells with their environment 34,54. The length

\footnotetext{
${ }^{7}$ here we mean by 'cell' the numerical discretization element and not the biological entity
} 
of microtubules is between 5 and 8 cell units $[76]$, whereas a glioma cell has a diameter of ca. $15 \mu \mathrm{m}=15 \times 10^{-3} \mathrm{~mm}$ [1. This means that the regions of MTs that would correspond to the tumor front have a length around $100 \mu \mathrm{m}=0.1 \mathrm{~mm}$. Then, the parameter $\gamma$ will represent the ratio of such MT area with respect to the whole tumor; here we take $\gamma=0.001$. Therefore, and in view of the above choice of $\varepsilon$, the parameter $\alpha$ is taken here of the order of $10^{3}$.

The coefficient functions involved in the simulations are summarized below:

For all scenarios: $\mathcal{F}(h, \rho, Q)=\gamma \frac{h}{h_{0}} \rho Q$,

$$
\begin{aligned}
& \lambda_{0}=\frac{\kappa f(Q)}{F A+f(Q)}, \quad \lambda_{1}=\frac{\kappa F A}{(F A+f(Q))^{2}}, \quad f(Q)=\frac{k_{+} Q}{k_{+} Q+k_{-}} \\
& f^{\prime}(Q)=\frac{k_{+} k_{-}}{\left(k_{+} Q+k_{-}\right)^{2}}, \quad g\left(Q, \lambda_{0}\right)=\frac{f^{\prime}(Q)}{k_{+} Q+k_{-}+\lambda_{0}}, \quad r_{D}(h)=r_{0}\left(\frac{h}{h_{0}}-1\right)_{+} .
\end{aligned}
$$

For Scenario 1: $Q(x)=1-\left(\frac{\operatorname{tr}\left(\mathbb{D}_{\mathbb{W}}(x)\right)}{\lambda_{\max }}\right)^{\frac{3}{2}}, \quad \mathbb{D}_{T}(x)=\frac{s^{2}}{(N+2)}\left(\mathbb{I}_{N}+2 \frac{\mathbb{D}_{\mathbb{W}}(x)}{\operatorname{tr}\left(\mathbb{D}_{\mathbb{W}}(x)\right)}\right)$,

\begin{tabular}{|c|c|c|c|}
\hline parameter & value & meaning & source \\
\hline$s$ & $50(\mu \mathrm{m} / \mathrm{h})(=1.389 \mathrm{e}-8 \mathrm{~m} / \mathrm{s})$ & cell speed & 67 \\
\hline$\alpha$ & $10^{3}(1)$ & weight for advective terms & estimated \\
\hline$\kappa$ & $0.1(1 / \mathrm{s})$ & maximum turning frequency & estimated \\
\hline$h_{0}$ & $1.0 \mathrm{e}-7.2(\mathrm{~mol} / \mathrm{l})$ & healthy acidity value & 84 \\
\hline$h_{T}$ & $1.995 \mathrm{e}-07(\mathrm{~mol} / \mathrm{l})$ & threshold of possible proliferation & 84 \\
\hline$D_{H}$ & $0.5 e-03\left(\mathrm{~mm}^{2} / \mathrm{s}\right)$ & diffusion coefficient of protons & 60 \\
\hline$a$ & $2.2 e-17\left(\mathrm{~mol} \mathrm{~cm}{ }^{3}(\text { cells } \cdot \mathrm{sec})^{-1}\right)$ & acid production rate & 65 \\
\hline$b$ & $0.8 \mathrm{e}-04(1 / \mathrm{sec})$ & proton buffering by healthy tissue & 64 \\
\hline$\eta$ & $0.26 \mathrm{e}-06(1 / \mathrm{sec})$ & tumor proliferation rate & $\overline{66}$ \\
\hline$k_{+}$ & $0.034(1 / \mathrm{sec})$ & attachment rate of tumor cells to tissue & 58 \\
\hline$k_{-}$ & $0.01(1 / \mathrm{sec})$ & cell detachment rate & 58 \\
\hline$\gamma$ & $5 \mathrm{e}-08(1 / \mathrm{sec})$ & acid-induced death rate of tumor cells & 81 \\
\hline$r_{0}$ & $1.0 \mathrm{e}-6(1 /(\mathrm{mol} \mathrm{sec}))$ & efficiency of fiber degradation & estimated \\
\hline
\end{tabular}

with $\lambda_{\max }$ denoting the maximum eigenvalue of the water diffusion tensor $\mathbb{D}_{W}$.

Table 1: Full set of parameters for the numerical simulations

\subsection{Numerical results}

We focus the presentation of the numerical results on those aspects where the new model and its extension, in particular by including tissue dynamics, produce new qualitative effects. Computations were conducted with a simulated time of 52 weeks. We first present line plots from the coordinate origin through the tumor to the upper right of the domain in order to visualize and discuss the evolution of the involved fields. This helps to establish the general dynamics of the model in Subsection 4.3.1. We present the final states of the involved fields in 4.3 .2 and investigate in Subsection 4.3 .3 which advective effect is playing a dominant role with respect to the tumor extent and appearance. We then directly compare the solutions of the three considered scenarios in Subsection 4.3.4, and address in Subsection 4.3.5 the grading of a tumor upon relying on the numerical results, particularly with respect to the amount of necrotic matter. We also suggest in Subsection 4.3.6 how the simulations of the model could be used to perform dose painting for radiation treatment.

\subsubsection{Scenario 3: overall dynamics}

Figure 1 shows the amplitude of the four fields $\rho, n, Q$ for densities of tumor cells, necrotic matter, and macroscopic tissue, respectively, and for the proton concentration field $h$ converted to its corresponding $p H$-value (right scale) at three distinct points in time. The uppermost plot indicates the initial condition we chose for the $\rho$ - and $h$ fields, progressing then downwards with the simulation time. 
As the tumor density $\rho$ increases due to the proliferation (thus also leading to an increase in acidity concentration) the cells start to accumulate at the interface with tissue due to $\mathrm{pH}$-taxis and meso- and macroscopic haptotaxis. The sustained expression of protons (lower $\mathrm{pH}$ ) leads to necrotic core formation and growth. The total neoplasm is considered to be made up by the densities of necrotic matter $n$ and living tumor cells $\rho$. At the final stage of the simulation, the former represents the largest part of the neoplasm $(n>0.6)$, with a comparatively small density (approx. $\rho<0.2$ ) of living tumor cells. This is consistent with high grade tumors typically observed in clinical practice 35,78 .

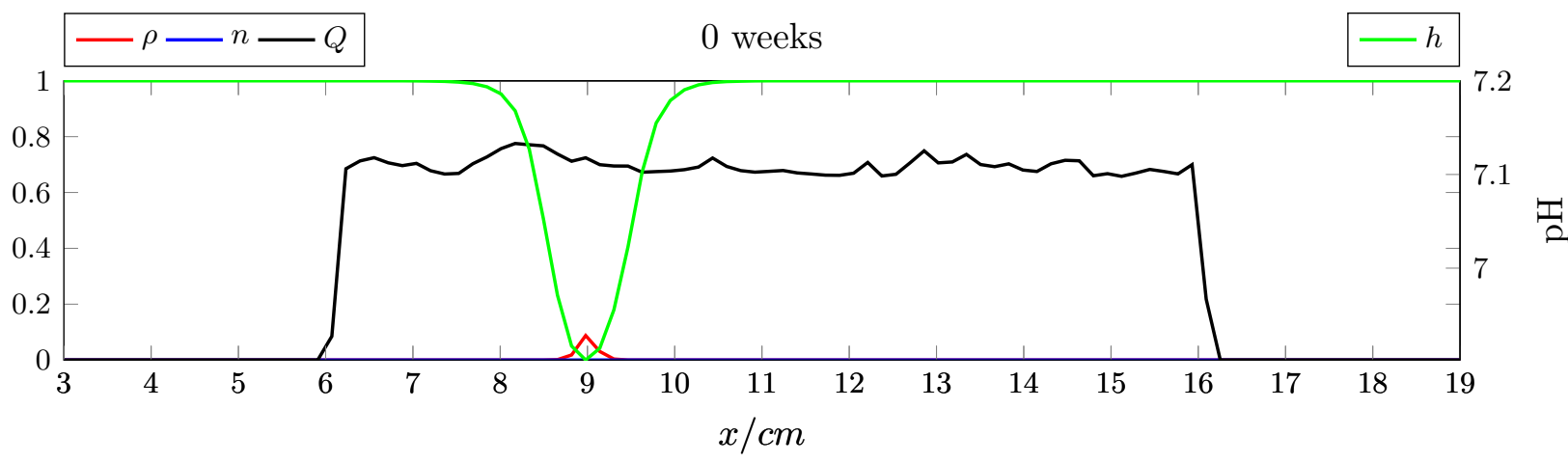

26 weeks

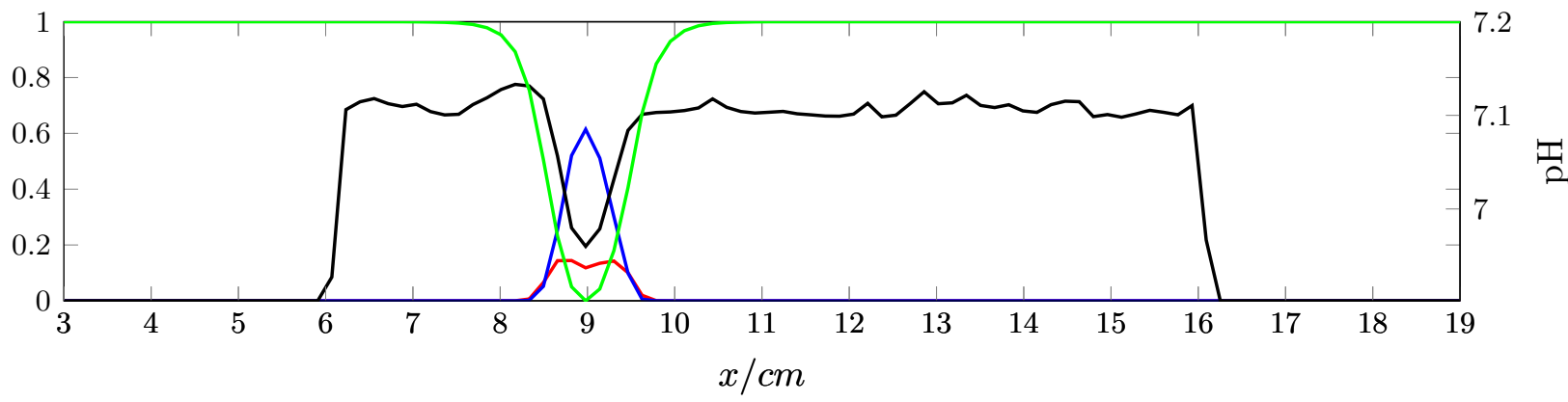

52 weeks

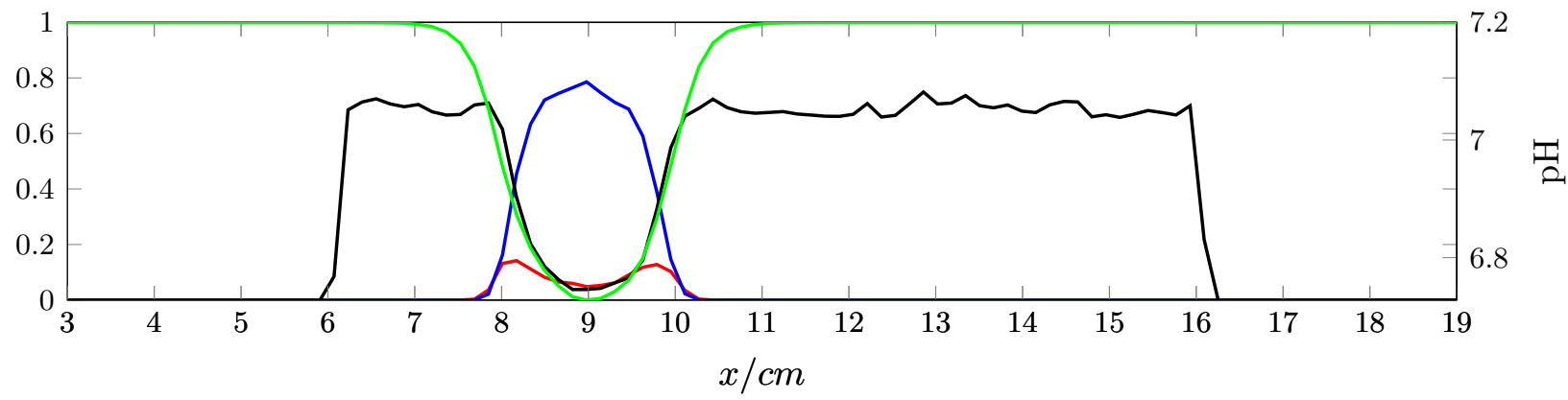

Fig. 1: Profiles of tumor density $\rho$, acidity $h$ (as $\mathrm{pH}$ ), necrosis $n$, and tissue density $Q$ over 52 weeks. Scenario 3: the tissue is dynamically degraded depending on its local orientation and the acidity concentration; a necrotic core is formed and develops, becoming the main part of the neoplasm. Available in color online.

\subsubsection{Scenario 3: solution components at the end of simulation}

In Figure 2 we show the final states of the four fields of Scenario 3, overlain with the main axis of the water diffusion tensor $\mathbb{D}_{w}$ from the DTI data sets, as well as the direction of the combined advective fields. The dominant $S_{2}$ term describing repellent $\mathrm{pH}$-taxis results in active transport of tumor mass away from the center. Similarly to the line plots presented earlier in Figure 1 , we can identify a reduction of active tumor cells and tissue degradation within the acidic core area of the neoplasm, where necrosis is growing instead. 

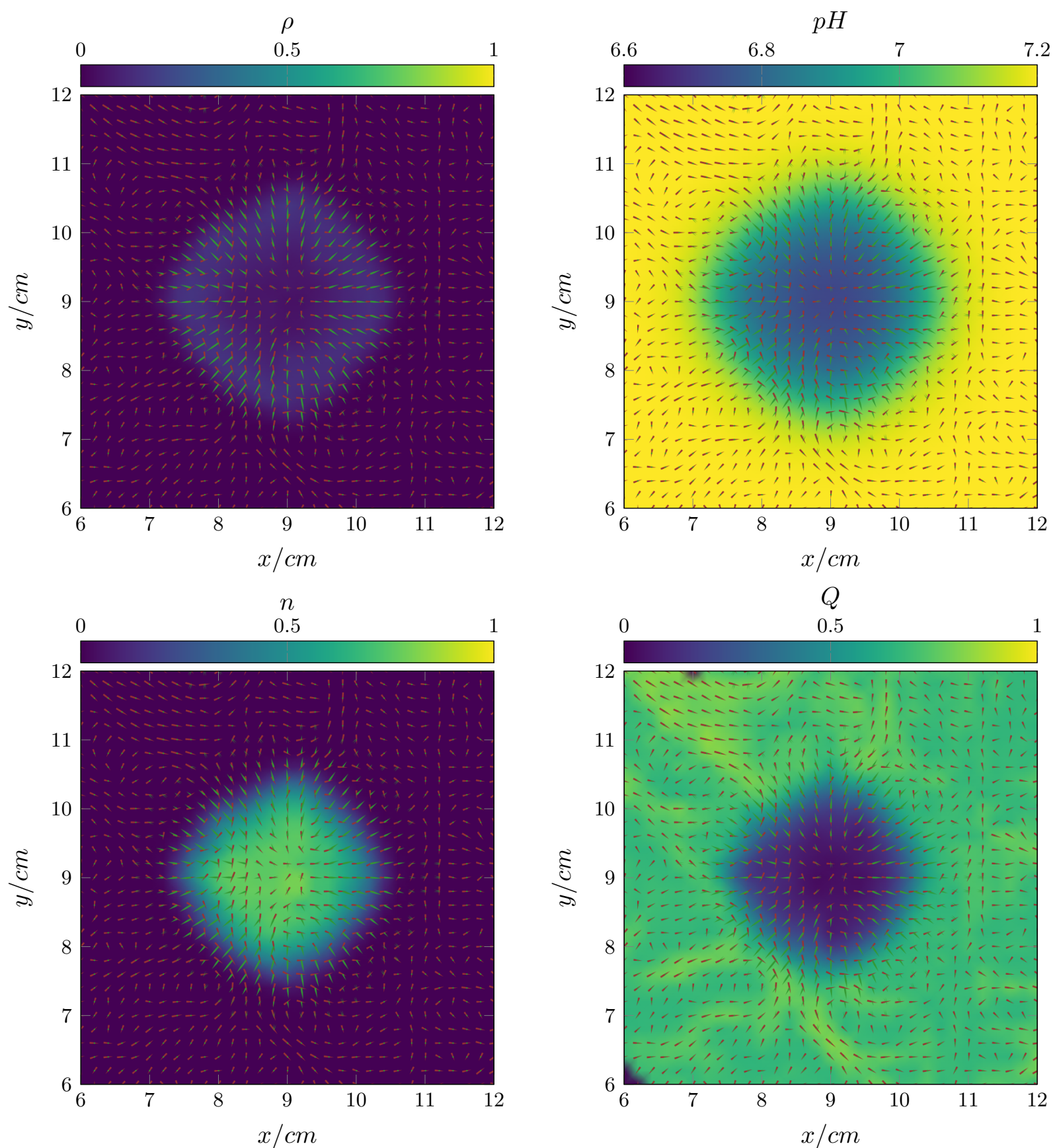

Fig. 2: Dynamic fields of solution components $\rho, p H=-\log _{10}(h), n, Q$ after 52 weeks in a computation for Scenario 3. Red arrows: main axes of $\mathbb{D}_{W}$. Green arrows: total advection vector. Top left: density $\rho$ of living tumor cells. Top right: $\mathrm{pH}$ value calculated from the acidity field $p H=-\log _{10}(h)$. Bottom left: density $n$ of necrosis. Bottom right: macroscopic density $Q$ of normal tissue. Available in color online. 


\subsubsection{Scenario 3: study of dominant advective effects}

To evaluate the relative importance of the four advective terms within the model, we investigated their dynamically changing magnitudes. We include the dynamic scaling term $1 / \lambda_{0}(x)$ in these presentations. Figure 3 shows the fractional anisotropy of the underlying data set, the magnitudes of $S_{1}, S_{2}$, and the magnitude of all combined advective terms at 52 weeks simulation time. For the literature-based parameter set in Table 1 the chemotactic term $S_{2}$ is orders of magnitudes stronger than the other advective fields in this late stage of tumor progression. In the bottom right plot of Figure 3 it can be observed that the dominant advective effect at the proliferation front is given by $S_{2}$, while the other effects may only contribute significantly in the absence of acidity gradients, i.e. beyond the visible tumor margins.

We also investigated in Figure 4 the relative importance of the different advective effects by successively excluding them in the simulation runs. That way, the total time-integrated effect of inclusion and exclusion of the terms could be investigated. The repellent $\mathrm{pH}$-taxis described by the $S_{2}$-term was found to have the most dominant effect on the tumor density, but the haptotaxis terms also contribute to establishing low-density areas at the tumor edges. Given the infiltrative spread of glioma cells and post-treatment tumor recurrence which is mainly due to such relatively small cell aggregates located further away from the main tumor mass, such margins might be relevant for treatment planning, especially as far as radiotherapy is concerned (see also Subsection 4.3.6.

\subsubsection{Comparison between model scenarios}

In the following we compare the simulation results for Scenarios 1-3 with identical initial condition, in order to find out whether there are any potentially relevant differences. Figure 5 shows the density $\rho+n$ of the neoplasm after 52 weeks. As expected, Scenario 3 involving three-fold taxis and myopic diffusion predicts the largest tumor spread, with the most substantial difference being that w.r.t. Scenario 1. It is a modeling problem in itself to predict how living cells and necrotic matter influence the gray scale images in different ways. A direct comparison of Scenarios 2 and 3 with medical images is therefore not possible without knowing the underlying mapping of the two species to the MRI gray scale imaging. However, the distinction of $\rho$ and $n$ allows deeper insights into the dynamics of the tumor expansion. The two plots in the second row of Figure 5 illustrate the differences between the densities $\rho+n$ after 52 simulated weeks, computed upon using Scenarios 3-2, and 3-1. Thus, the latter comparison shows that taking into account the evolution of normal tissue is of high relevance, while considering the dynamics of mesoscopic tissue distribution $q$ (i.e the step from Scenario 2 to 3 ) is contributing less (please note the different scales of the color maps therein). Hence, the evolution of macroscopic tissue density $Q$ seems to contribute most to the prediction of tumor spread.

On the other hand, the dynamics of mesoscopic tissue $q$ contributes to substantially modifying the fractional anisotropy FA, which is a key observable in the DTI imaging of brain tumors. Our new model allows to directly model the depletion of brain structure and the therewith associated changes in FA, as indicated in Figure 6. The two plots of the first row show the initial fractional anisotropy and how it is diminished by the tumor growth after 52 weeks, again overlain by the direction of the total advective fields. The plot on the second row illustrates the difference in FA between the final and the initial simulation times.

The results indicate that tissue dynamics can be seen as a downstream effect, after tumor density and acidity have changed from baseline. The differences in $F A$ are therefore most prominent closer to the tumor center, and not so much at the invasion edge. Scenario 3 is more complex and requires more effort to treat, but beyond the differences put in evidence in Figures 5 and 6 it also provides a framework for a careful description of macroscopic tissue dynamics - along with its effects on the other solution components.

Still with the aim of investigating the influence of evolving tissue, we also compare a reduced version of Scenario 3 with a previous model obtained in $[72$ and accounting for the effect of tissue anisotropy on the migration of glioma cells, however with fixed tissue. Specifically, we compare the PDE

$$
\rho_{t}=\nabla \nabla:\left(\frac{1}{\lambda_{0}} \mathbb{D}_{T} \rho\right)+\eta \rho(1-\rho)
$$

which is a slight modification of that in 72 , by adding the proliferation term and letting $\lambda_{0}$ be 

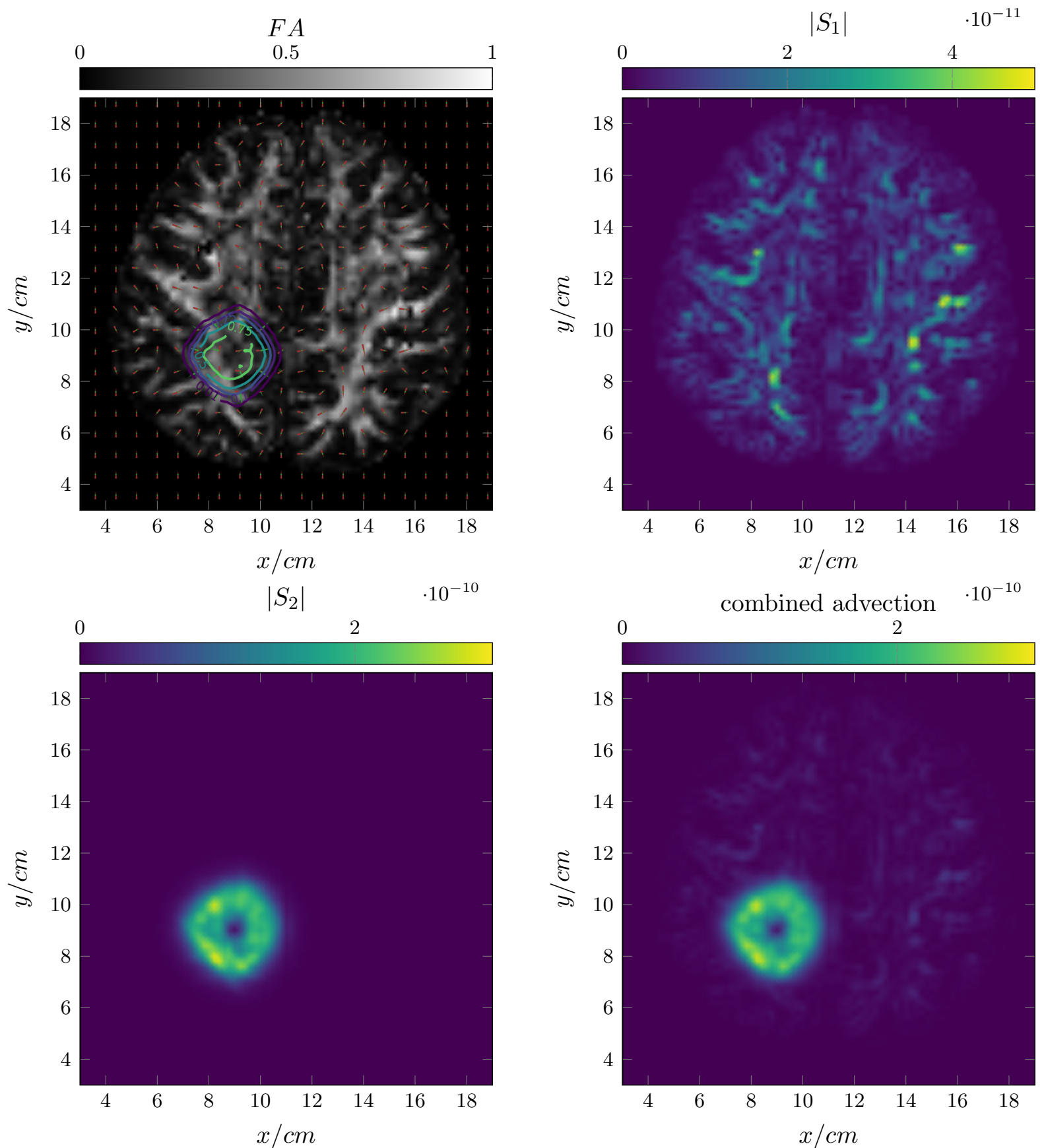

Fig. 3: Top left: fractional anisotropy of the camino dataset 17. Contours: neoplasm density $\rho+n$ from Scenario 3 after 52 weeks. Red arrows: main axis of $\mathbb{D}_{W}$. Green arrows: total advection vector. Top right: magnitude of the $S_{1}$ term. Bottom left: magnitude of the $S_{2}$ term. Bottom right: magnitude of all advective terms (myopic drift, macroscopic haptotaxis, mesoscopic haptotaxis $S_{1}$, repellent $\mathrm{pH}$-taxis $S_{2}$ ). The scales of the color maps differ between the plots. All advective terms contain the scaling factor $1 / \lambda_{0}(x)$. Available in color online. 
scenario 3: neoplasm: $\rho(x)+n(x)$, all adv. fields, 52 weeks

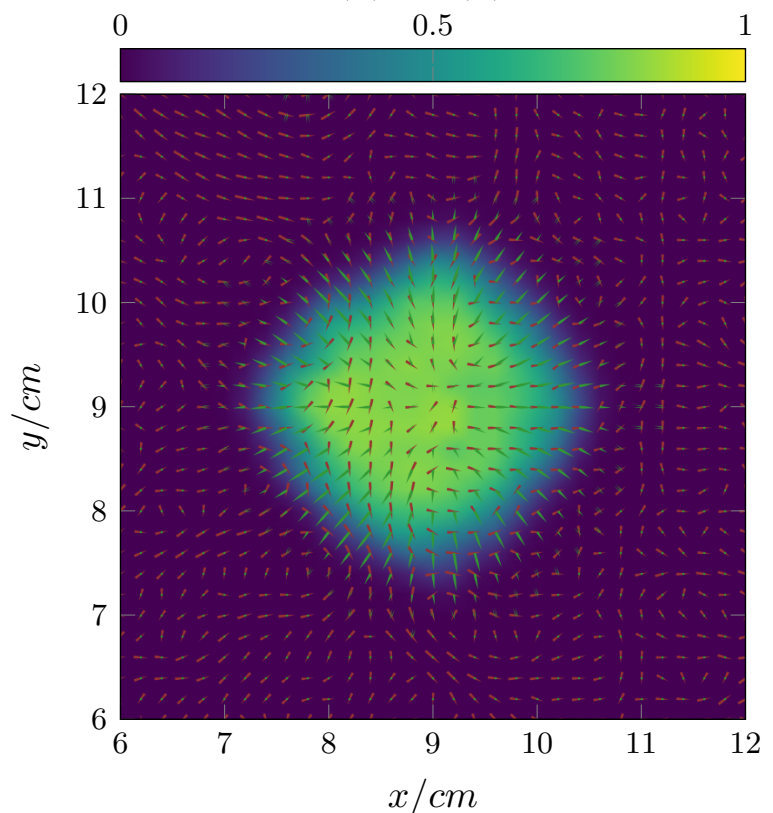

scenario 3 no $S_{1}$

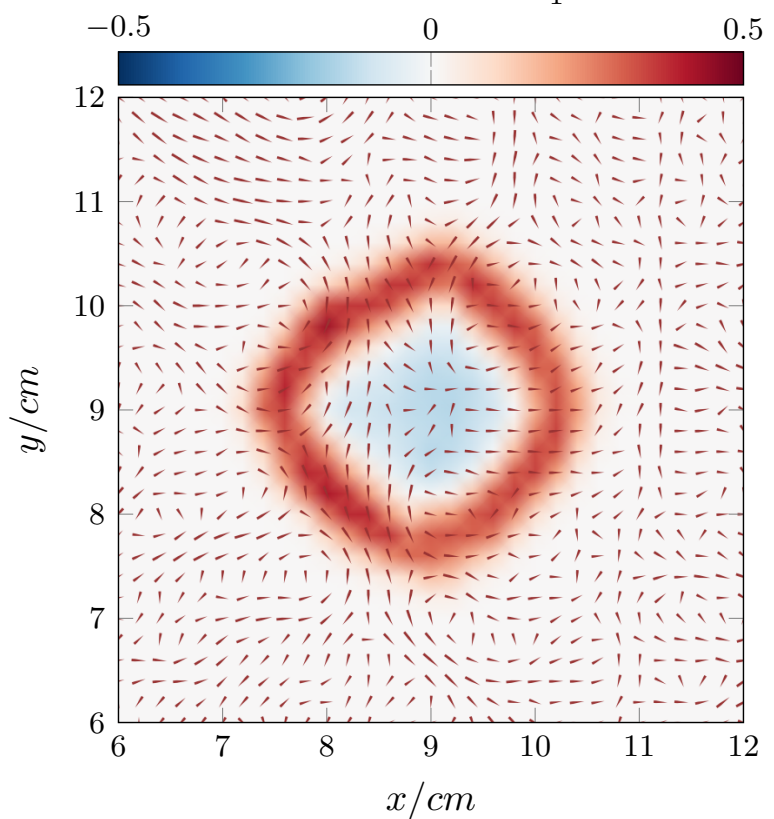

scenario 3: no haptotaxis $\cdot 10^{-4}$
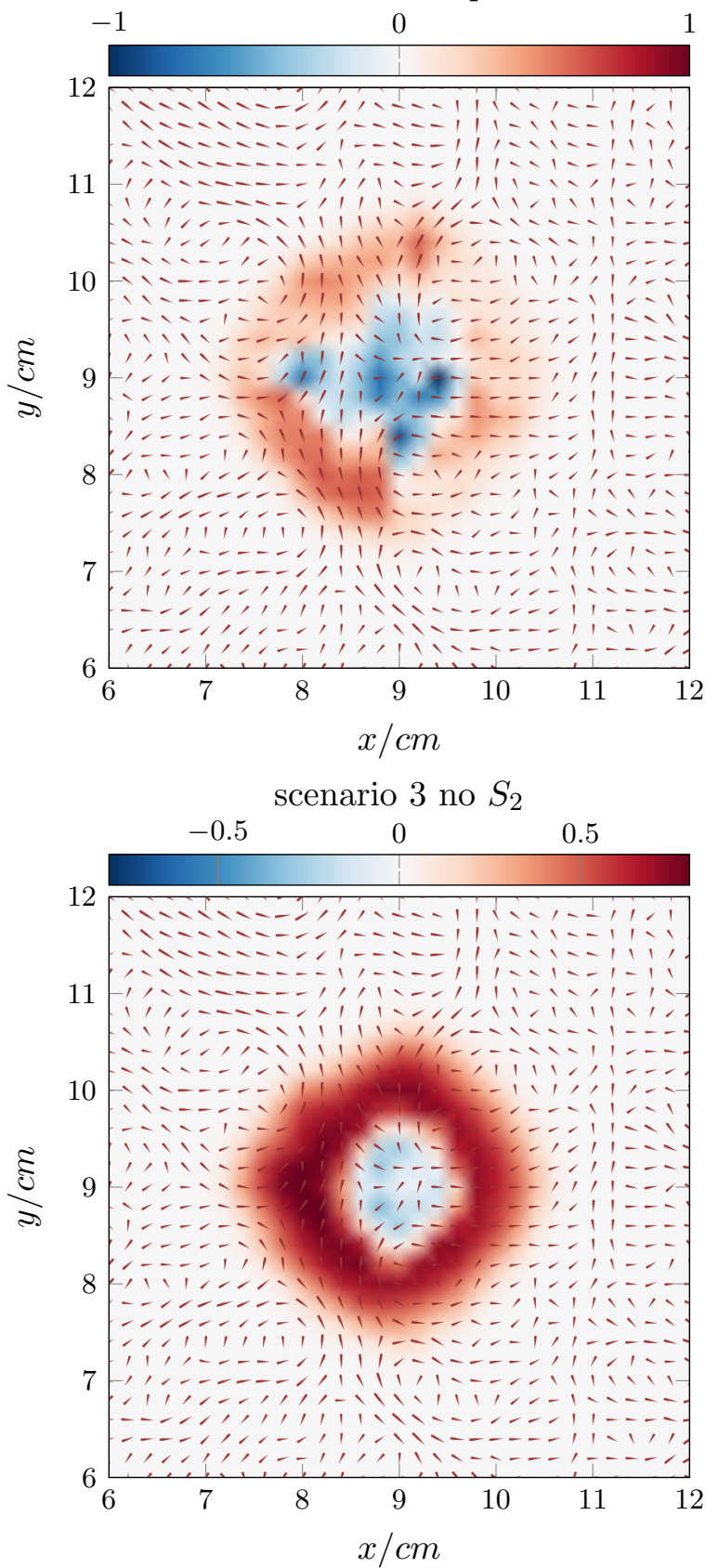

Fig. 4: The effect of advective fields on the model predictions. Red arrows: main axis of $\mathbb{D}_{W}$. Green arrows: total advection vector. All results are obtained for Scenario 3. Top left: simulation result for the density $\rho+n$ of the neoplasm after 52 weeks. The rest of the plots show absolute differences in $\rho+n$ between the full model and Scenario 3 without one of the adective terms. Top right: no macroscopic haptotaxis. Bottom left: no $S_{1}$ advection. Bottom right: no $S_{2}$ advection. The scales of the color maps differ between the plots. Available in color online.

nonconstant, but depend on $x$ like in the rest of this paper, with the system

$$
\begin{aligned}
& \rho_{t}=\nabla \cdot\left(\frac{1}{\lambda_{0}} \nabla \cdot\left(\mathbb{D}_{T} \rho\right)-\lambda_{1} g\left(Q, \lambda_{0}\right) \mathbb{D}_{T} \nabla Q \rho-\alpha S_{1}(q) \rho\right)+\eta \rho(1-\rho) \\
& q_{t}=r_{0} \rho q\left(\Pi_{a}[q]-A[q]\right) \\
& Q_{t}=r_{0} \rho Q(A[q]-1),
\end{aligned}
$$



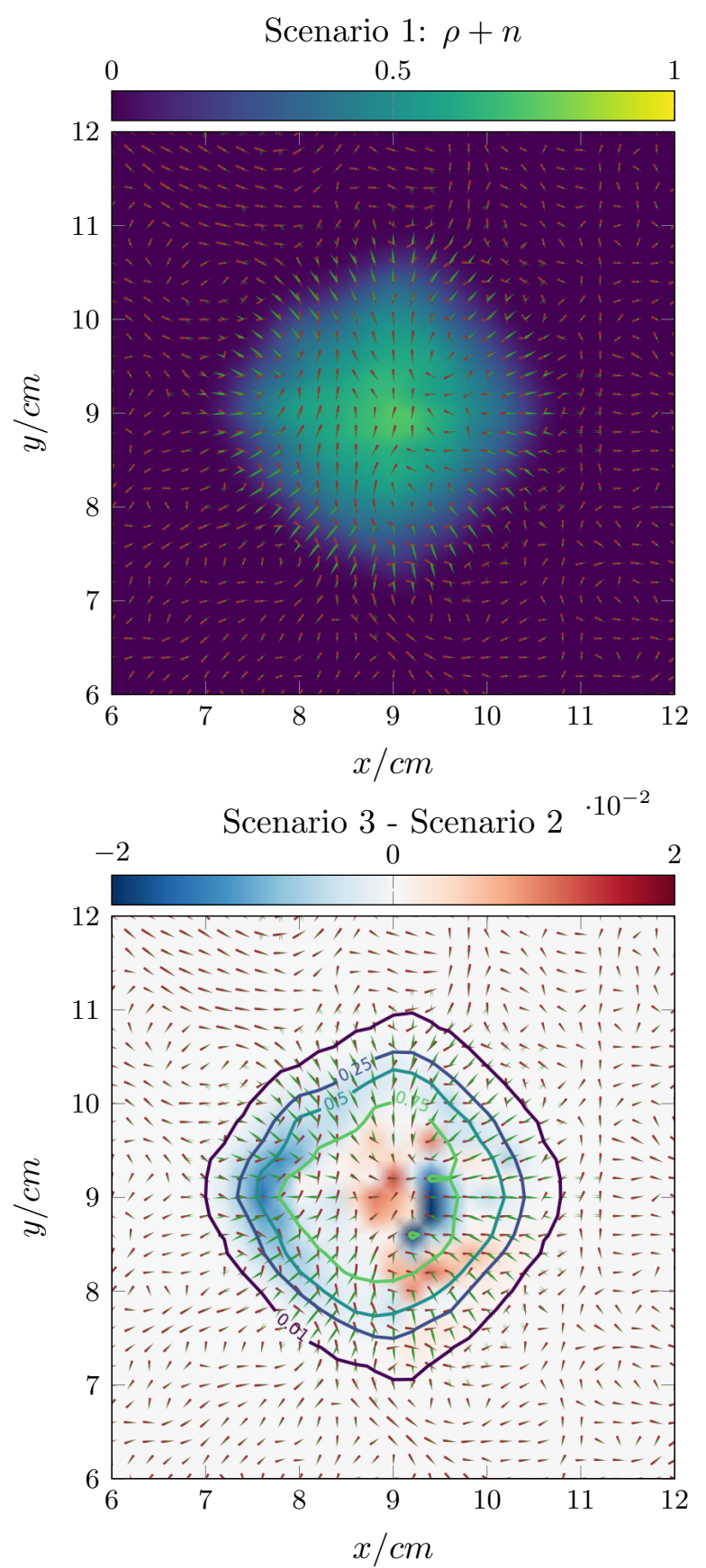
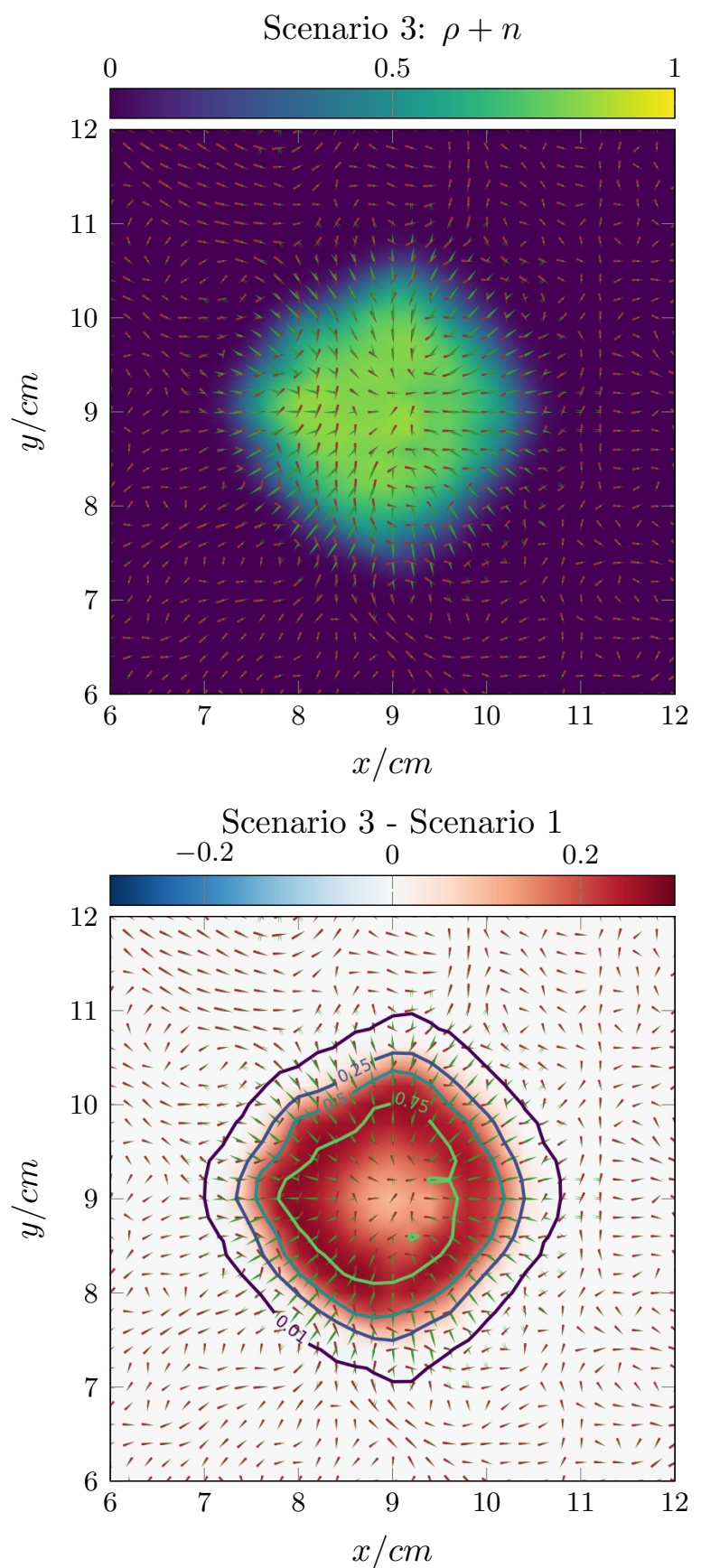

Fig. 5: Comparison of the scenarios. Contours: density $\rho+n$ of the neoplasm computed in the framework of Scenario 3. Red arrows: main axis of $\mathbb{D}_{W}$. Green arrows: total advection vector. Top row: density $\rho+n$ at the final time (52 weeks) for Scenarios 1 and 3. Bottom row: pointwise difference in $\rho+n$ between Scenarios 3 and 2 and difference between Scenarios 3 and 1. Note: the scales of the color maps differ between the plots. Available in color online. 
both with the same initial conditions and no-flux boundary conditions and the same function $\lambda_{0}$. Figure 4.3 .4 shows the computation results. Note that none of the two model makes any distinction between active and necrotic tumor. The complete tumor mass is encoded in $\rho$. As before, the evolution of tissue leads to substantial changes in FA, although the dynamics of acidity and its influence are absent in these settings. The simulations predict a larger area of glioma spread when the cells are allowed to degrade the tissue; although there are rather small amounts of tumor cells in the enlarged regions, these might be relevant for tumor recurrence. Model 4.54) leads to higher cell densities at the core of the neoplasm, due to the migrating cells only performing myopic diffusion and not being supplementary driven by haptotaxis.

\subsubsection{Tumor grading and importance of tissue evolution}

Tumor grade assessment is decisive for the treatment planning of glioma and for patient survival prognosis. Typically, glioma are classified according to cell activity and tumor aggressiveness, upon relying on a series of indicators, including histological patterns 14,89 and tumor composition. Among these, grading by the amount of necrosis relative to the whole tumor volume (both visible via biomedical imaging) has been addressed e.g., in [35, 55, 78]. In fact, [35] observed that 'location and volume of tumors were not statistically significant predictors of survival', which is in accordance to most conducted studies, as reviewed e.g. in [37]. We therefore address here only necrosis-based grading. In 35 the percentage of necrotic matter visible on MRI is related to the total visible tumor volume. An attempt at tumor grading by way of connecting the results of numerical simulations for our model to this definition proves to be difficult without in-depth information on how the tissue types (e.g., obtained by segmentation) affect the MRI shading. The numerical results of our model do not contain any thresholding or shading effects, therefore we define the time-dependent grade $G(t) \in[0,1]$ of the simulated tumors via:

$$
G(t):=\frac{V_{n}(t)}{V_{n}(t)+V_{\rho}(t)}
$$

where $V_{n}(t)$ and $V_{\rho}(t)$ denote integrals over the whole space domain $\Omega$ of the necrosis and living cell densities $n(t, x)$ and $\rho(t, x)$, respectively. We investigate then the evolution of this quantity over time, upon being guided by the percentage classification in 35]: $0<G<25 \%$ : grade 1; $25 \% \leq G<50 \%$ : grade 2; $G \geq 50$ : grade 3, with the highest grade corresponding to the most aggressive tumor and the poorest prognosis.

Figure 8 illustrates the evolution of the tumor grade for the three considered scenarios. We found only tiny differences between the grades for Scenarios 2 and 3, whereas the difference in tumor grade progression between Scenarios 1 and 2 is substantial. The definition (4.56) of grade $G$ effectively skips grade 0 , as there is no threshold on the visibility of the necrotic tissue density $n$. The fact that Scenario 1 fails to predict tumor progression into grade 3 in due (biomedically realistic) time might be an indicator of Scenario 1 being insufficient for modeling glioma dynamics, thus endorsing the role played by tissue evolution.

\subsubsection{Dose painting}

Beyond tumor grading based on assessment of necrotic matter and heterogeneity of the neoplasm, the model can provide valuable information about the extent and shape of the tumor and hence help establishing the contours for radiation treatment. The ability to predict space-time densities of clonogens as well as necrosis opens the way for more accurate tumor segmentation and dose painting. Figure 9 shows a gray scale image of the computed neoplasm $(\rho+n)$ for a simulated time of 52 weeks. Level sets for the density of living glioma cells are shown, thus providing information about the regions where higher radiation doses should be applied in order to eradicate the active cells, at the same time sparing areas where their density is lower (either as a consequence of necrosis - in the tumor core, or due to less cells having invaded the healthy tissue at the tumor margins).

\section{Discussion}

We proposed in this paper a novel model for glioma invasion, which takes into account the interaction with the biochemical and biophysical tumor microenvironment, represented here by 

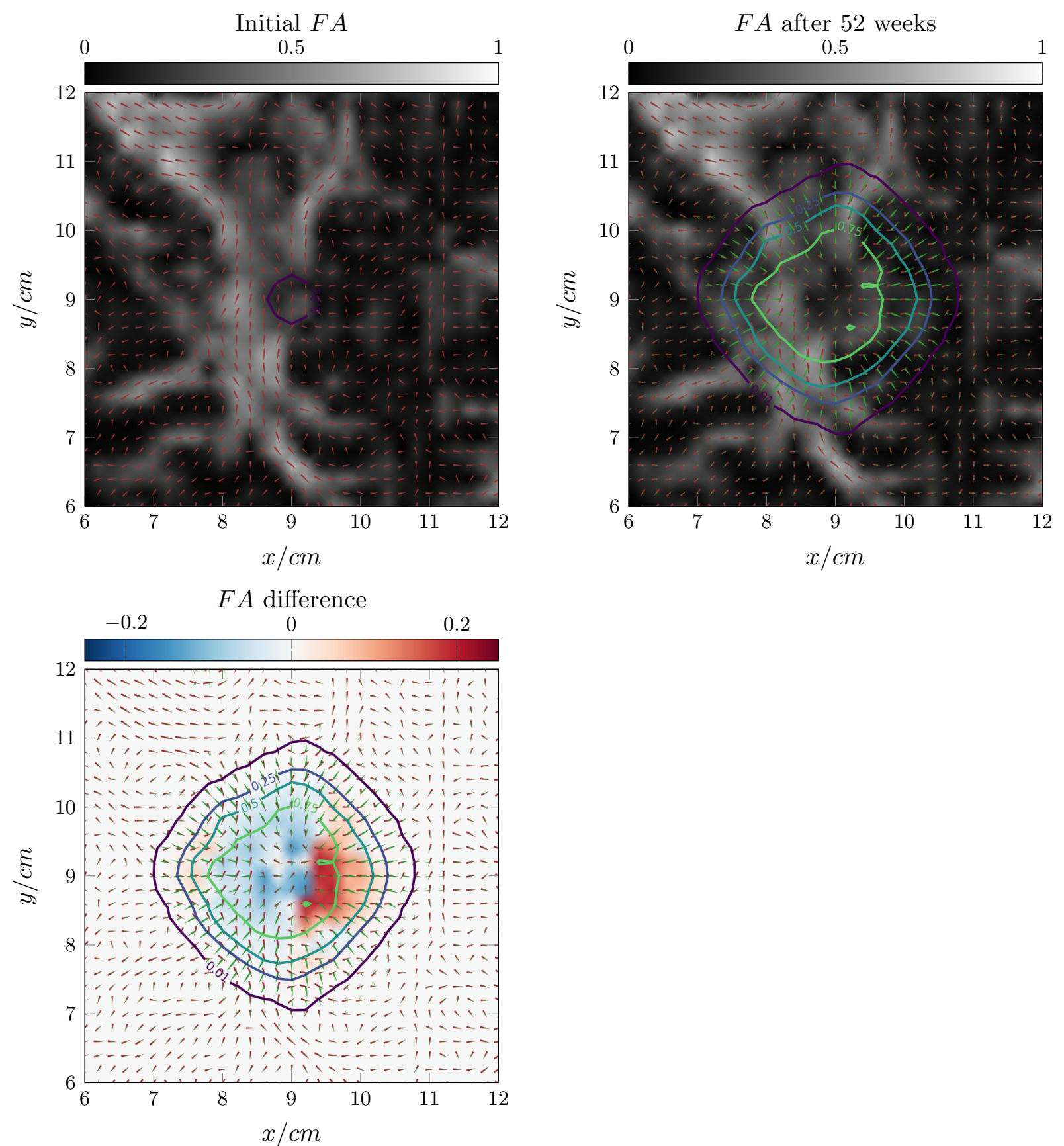

Fig. 6: Tissue dynamics in Scenario 3. Contours: neoplasm density $\rho+n$ from Scenario 3. Red arrows: main axis of $\mathbb{D}_{W}$. Green arrows: total advection vector. Top left: gray scale plot of $F A$ at the start of the simulation. Top right: $F A$ after 52 weeks, with visible reduction of anisotropy closer to the tumor centre. Bottom left: difference in $F A$ between final and initial simulation times. Available in color online. 

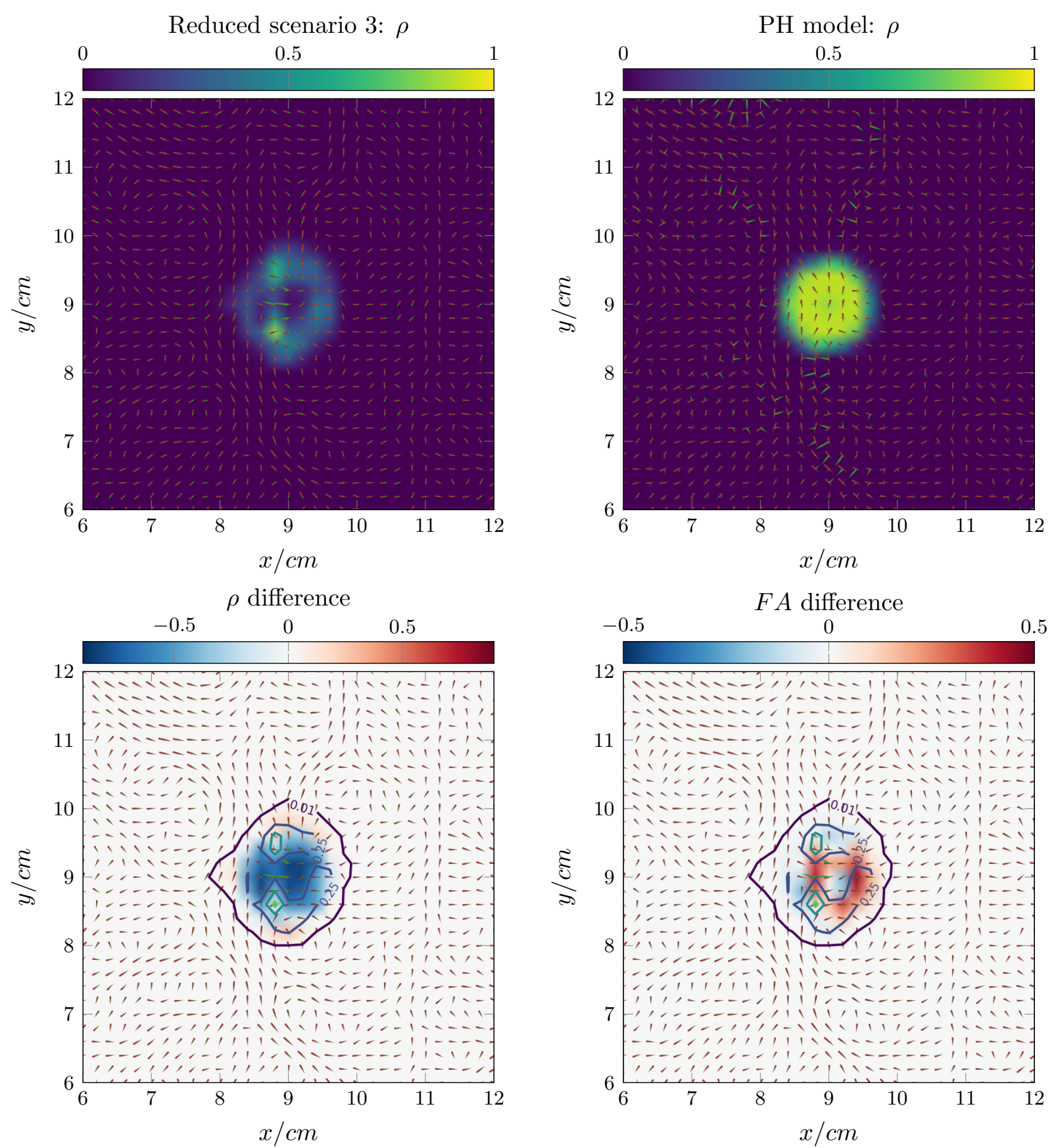

Fig. 7: Comparison between models (4.55) and (4.54). Contours: tumor density $\rho$ from the computation of 4.55) (purple: $\rho=0.1$, blue: $\rho=0.25$, green: $\rho=0.5$ ). Red arrows: main axis of $\mathbb{D}_{W}$. Green arrows: total advection vector. Top left: tumor density $\rho$ at the final time (52 weeks) for model (4.55). Top right: tumor density $\rho$ for model (4.54). Bottom left: difference in $\rho$ computed with model (4.55) and with model 4.54). Bottom right: Difference in $F A$ between end and beginning of simulation, computed with model (4.55). Available in color online. 
Numerical tumor grading for Scenarios 1-3

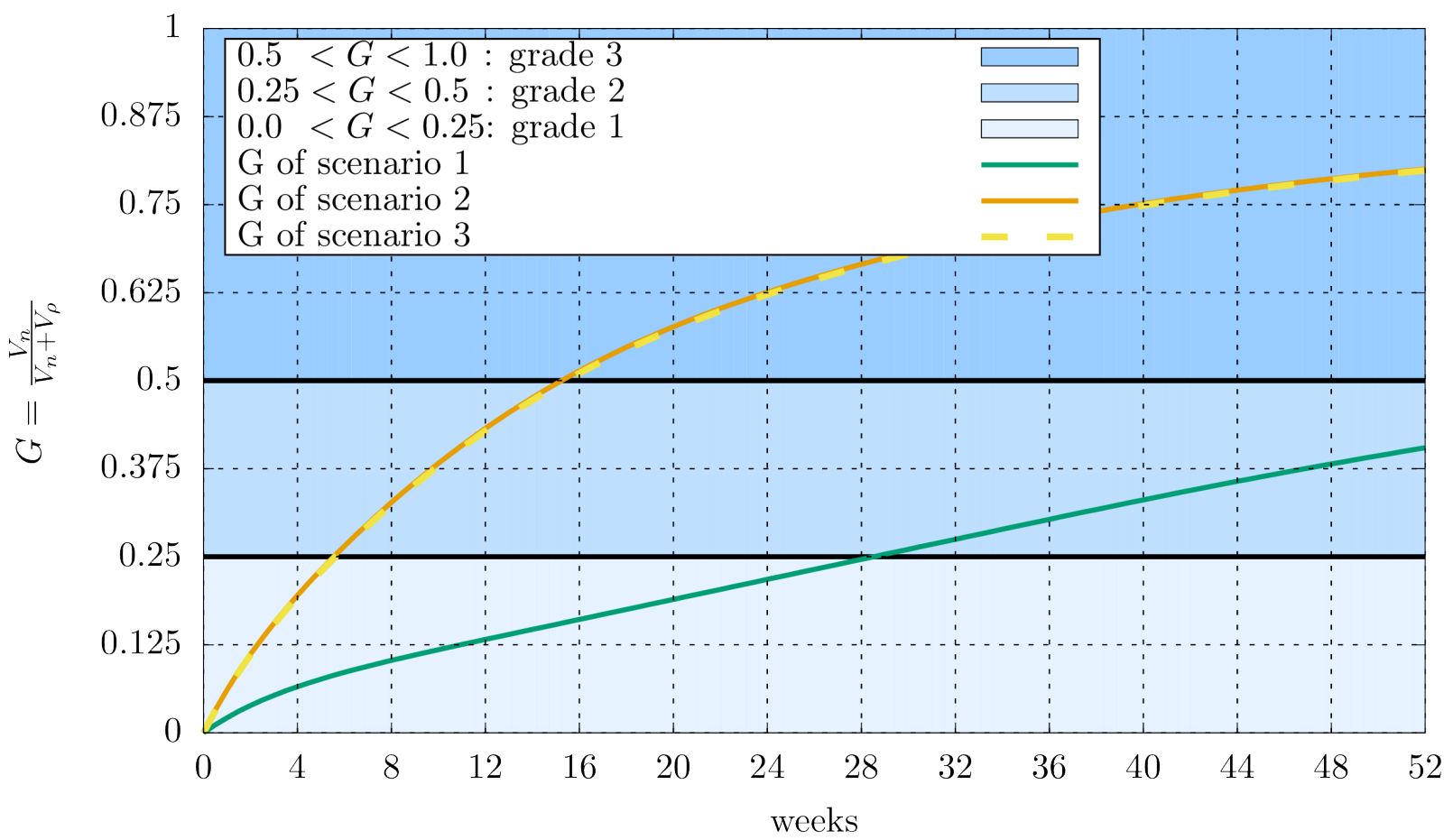

Fig. 8: Grade of tumor 4.56) for the three considered Scenarios. $G$ is essentially the same for Scenarios 2 and 3, whereas in Scenario 1 (with the same parameter choice) it exhibits significantly slower progress. Full information is assumed, so that there is no visibility threshold. Scenario 1 predicts the progression from grade 1 towards grade 2 after about 28 weeks, whereas in Scenarios 2 and 3 it needs only about 5 weeks. The progression to grade 3 occurs after about 16 weeks for Scenarios 2 and 3. For Scenario 1 it does not happen within the simulated time of 52 weeks (recall that the median survival time of patients with glioma is approx. 60 weeks 91 ). 


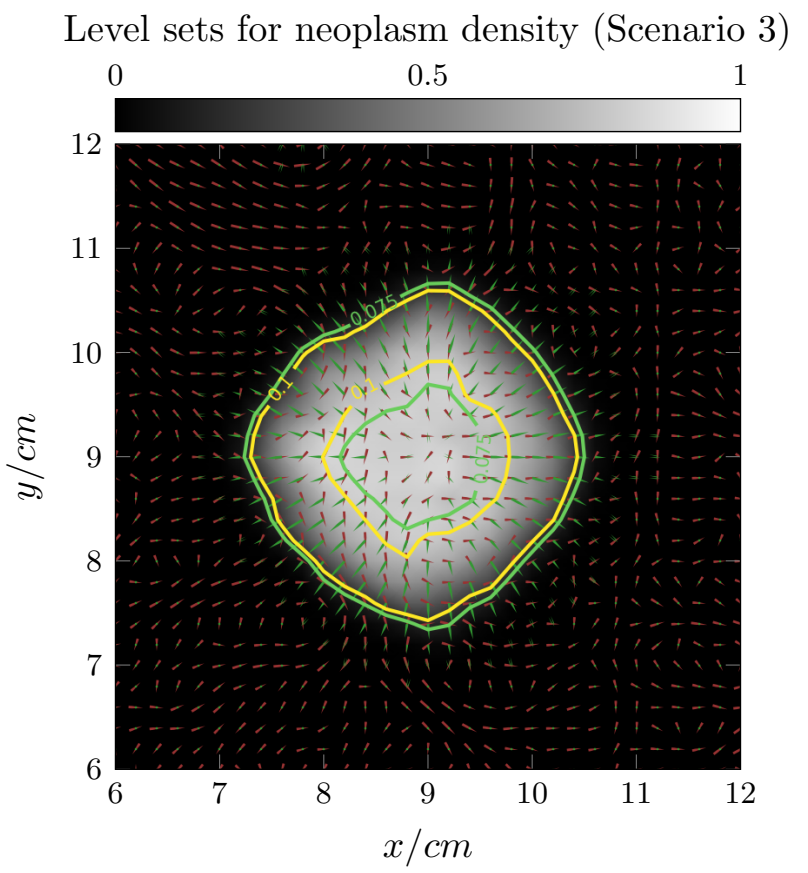

Fig. 9: Level sets for density $\rho$ of living tumor cells could be used for dose painting in radiotherapy. Gray scale image: density of neoplasm $\rho+n$, computation from the reduced Scenario 3, simulation of 52 weeks. Level sets: $\rho=0.1$ (yellow) and $\rho=0.075$ (green).

acidity acting as a repellent and, respectively, oriented tissue fibers guiding the cell migration. Both environmental components, most prominently the acidity, also influence the growth or depletion of tumor cells.

The modeling approach has a multiscale character: it starts from ODE descriptions of single cell (position, velocity, activity variable) dynamics, which are translated into a kinetic transport equation for the space-time distribution function of cells sharing the same regimes of velocity and activity variables, and eventually arrives at a macroscopic PDE for the evolution of the whole tumor as a population of such cells performing myopic diffusion and multiple taxis. The cell behavior is thereby dynamically coupled to that of the environment, the latter featuring a system of macroscopic and mesoscopic (integro-)differential equations with dependencies on space, time, and orientation of tissue fibers. We refer to 52 for a review of existing models of cell migration with multiple taxis and therewith associated challenges. The diffusion and taxis coefficients of the PDE obtained here for the cell density and the source terms of the equations for meso- and macrocopic tissue and necrosis densities involve nonlocalities w.r.t. the orientations of tissue fibers and cell velocities; see 20 for a review of models for cell migration involving this and other types of nonlocality.

There are several alternative ways to include cell level environmental influences in a KTAP modeling framework, ultimately leading in the macroscopic limit to taxis terms:

(i) by using in the operator describing velocity reorientations turning rates which depend on the pathwise gradient of one or several signals, as done e.g. in 63.70;

(ii) by describing the dynamics of activity variables for the cell state, which translates into corresponding transport terms in the kinetic equations and turning rates depending on the activity variable(s), see $23,25,27,41,42$ for glioma interacting with tissue and e.g., 28,92 for swimming bacteria biased by some chemoattractant;

(iii) by accounting for biochemical and/or biophysical effects translated into cell stress and forces acting on the cells and leading to transport terms w.r.t. the velocity variable in the kinetic PDE on the mesolevel, see 19] for the case of cancer cells responding to a 'chemotaxis force' proportional to the gradient of a given chemoattractant concentration;

(iv) by considering turning operators where the cell reorientation depends on the interaction with (some of) the environmental cues and using equilibrium distributions for the moment closure, as e.g. in [19]. 
While (i) and (iv) have a rather mesoscopic flavor, (ii) and (iii) refer closer to microscopic, single cell descriptions, therefore we employed in this work a combination of the latter: (ii) led to the macroscopic haptotaxis term, while (iii) with the description of cell stress and associated, speedpreserving forces acting on glioma cells yielded the terms characterizing repellent $\mathrm{pH}$-taxis and mesoscopic haptotaxis. Notice that the coupling with evolving acidity and macro- and mesoscopic tissue led to genuine taxis, unlike previous models $23,25,27,41,42$ where the tactic cue (e.g., macroscopic tissue) was only space-dependent. To our knowledge dynamics of such complexity has not been treated in previous works, neither numerically nor from an analytical viewpoint. In 71 were performed interesting simulations on the kinetic level for a simpler model of cells migrating through the extracellular matrix with oriented fibers, which they degrade according to certain rules. Our closed macro-meso description (3.35) has the advantage of a lower dimensional phase space, thus allowing -among others- to accommodate even further possibly relevant biological effects with a rather detailed description via (ii) and/or (iii), without facing problems due to a prohibitive increase of the number of kinetic variables and therewith associated computational costs.

Concerning possible relationships between (i)-(iv), it has been proved in 73 that (ii) actually implied (iii) under certain assumptions on the subcellular dynamics for a model of bacterial run-and-tumble swimming. In 57 such connection was investigated in a less rigorous way for a model describing glioma pseudopalisade patterning under the influence of repellent $\mathrm{pH}$-taxis and anisotropic, but non-evolving tissue.

In order to pass from the KTAP framework to effective equations for the tumor spread we performed here a hyperbolic as well as a parabolic upscaling, according to the underlying tissue being directed or not. The latter is still not clarified from a biological viewpoint; the study (via numerical simulations) performed in [57] for the mentioned model of glioma pseudopalisades revealed that such patterns which are invariably observed on histological samples of high grade tumors 14,89 formed in due time when using the system obtained in the parabolic limit, while the hyperbolic scaling led to a drift-dominated system predicting only a rapid shift of the initial aggregate of glioma cells towards the leading tissue orientation, without pattern development. This suggests that brain tissue might be undirected and induced us here to perform numerical simulations only for the equations obtained by parabolic scaling.

Our model makes use of DTI data and accounts for the evolution of brain tissue, transferring biomedical information through the directional distribution of tissue fibers. As mentioned before, this information belongs to both micro and macro worlds and they coexist in the macroscopic limit. In Subsection 3.1 we put in evidence the versatility of this model w.r.t. the influence of (spatially varying) tissue anisotropy in characterizing the tumor spread, thereby also considering the impact of hypoxia. In fact, our study of dominant advective effects suggests that $\mathrm{pH}$-taxis is the dominating drift - at least as far as this model and its parameterization are concerned. In this work we used the approaches (ii) and (iii) to describe cell-tissue interactions and (iii) for the response of cells to acidity. This led to the anisotropy-triggered switch between myopic diffusion with 'meso-haptotaxis' and the migratory behavior with the supplementary 'macrohaptotaxis'. Instead, using (ii) for cell-acid interplay and correspondingly define the turning rate would conduct to a hypoxia-triggered switch. Several other options of combining (ii) and/or (iii) can be conceived as well, raising further questions about the relationship of these alternative approaches. How appropriate a particular model is for a specific problem eventually depends on a multitude of factors and can properly be assessed when validated with adequate patient data. The current high costs of collecting such data still keeps a reliable validation out of reach, but the unprecedented development of biomedical technology during the last decade gives hope of it becoming available in the near future.

\section{Acknowledgement}

GC, CE, AK, CS, and MW acknowledge funding by the Federal Ministry of Education and Research BMBF in the project GlioMaTh 05M2016. This work has been also partially supported by the MINECO-Feder (Spain) research grant number RTI2018-098850-B-I00, the Junta de Andalucía (Spain) Project PY18-RT-2422 \& A-FQM-311-UGR18 (JN, JS). We also thank our cooperation partners C. Berdel, Y. Dzierma, S. Knobe, W. Reith, and C. Rübe from the Saarland University Hospital for their support and interesting meetings and discussions on glioma cancer and its treatment. 


\section{References}

[1] https://bionumbers.hms.harvard.edu/bionumber.aspx?s=n\&v=0\&id=108941.

[2] P. Basser, Diffusion and diffusion tensor MR imaging:fundamentals. Magnetic Resonance Imaging of the Brain and Spine (S. Atlas ed.). Philadelphia: Lippincott Williams, 2008, pp. $1752-1767$.

[3] P. Bastian, M. Blatt, A. Dedner et al, A generic grid interface for parallel and adaptive scientific computing. Part I: abstract framework. Computing 82 (2008) 103-119.

[4] P. Bastian, M. Blatt, A. Dedner et al, A generic grid interface for parallel and adaptive scientific computing. Part II: implementation and tests in DUNE. Computing 82 (2008) $121-138$.

[5] P. Bastian, F. Heimann, S. Marnach, Generic implementation of finite element methods in the Distributed and Unified Numerics Environment (DUNE). Kybernetika 46 (2010), 294-315.

[6] N. Bellomo. Modeling Complex Living Systems. Birkäuser, Boston, 2008.

[7] N. Bellomo, A. Bellouquid, J. Nieto, J. Soler, On the asymptotic theory from microscopic to macroscopic growing tissue models: an overview with perspectives. Math. Models Methods Appl. Sci. 22 (2012), 1130001, 37 pages.

[8] A. Bellouquid, J. Calvo, J. Nieto, J. Soler, Hyperbolic vs parabolic asymptotics in kinetic theory towards fluid dynamic models, SIAM J. Appl. Math. 73(4) (2013) 1327-1346.

[9] S. Benedetto, R. Pulito, S. Geninatti Crich, G. Tarone, S. Aime, L. Silengo, J. Hamm, Quantification of the Expression Level of Integrin Receptor $\alpha_{v} \beta_{3}$ in Cell Lines and MR Imaging With Antibody-Coated Iron Oxide Particles. Magnetic Resonance in Medicine 56 (2006) 711-716.

[10] T. Beppu, T. Inoue, Y. Shibata, A. Kurose, H. Arai, K. Ogasawara, A. Ogawa, S. Nakamura, H. Kabasawa, Measurement of fractional anisotropy using diffuson tensor MRI in supratentorial astrocytic tumors. J Neuro Oncol 63 (2003) 109-116.

[11] M. Blatt, P. Bastian, The Iterative Solver Template Library. Proc. of the 8th intern. conf. on Applied Parallel Computing: State of the Art in Scientific Computing, 2007, 666-675.

[12] M. Blatt, A. Burchardt, A. Dedner, Ch. Engwer, J. Fahlke, B. Flemisch, Ch. Gersbacher, C. Gräser, F. Gruber, Ch. Grüninger, D. Kempf, R. Klöfkorn, T. Malkmus, S. Müthing, M. Nolte, M. Piatkowski, O. Sander, The Distributed and Unified Numerics Environment, Version 2.4. Archive of Numerical Software, 2016.

[13] P. Bondiau, O. Clatz, M. Sermesant, P. Marcy, H. Delignette, M. Frenay, N. Ayache, Biocomputing: numerical simulation of glioblastoma growth using diffusion tensor imaging. Phys. Med. Biol., 53 (2008) 879-893.

[14] D. J. Brat, A. A. Castellano-Sanchez, S. B. Hunter, M. Pecot, C. Cohen, E. H Hammond, S. N Devi, B. Kaur, E. G. Van Meir, Pseudopalisades in glioblastoma are hypoxic, express extracellular matrix proteases, and are formed by an actively migrating cell population, Cancer Research 64 (2004) 920-927.

[15] T.A. Brunner, J.P. Holloway, Two-dimensional time dependent Riemann solvers for neutron transport. J. comp. Phys. 210 (2005) 386-399.

[16] L. Calorini, S. Peppicelli, and F. Bianchini, Extracellular acidity as favoring factor of tumor progression and metastatic dissemination. Exp. Oncol. 34 (2012) 79-84.

[17] Camino: Open-Source Diffusion-MRI Reconstruction and Processing, 14th Scientific Meeting of the International Society for Magnetic Resonance in Medicine, 2006.

[18] J. Chiche, M.C. Brahimi-Horn, J. Pouysségur, Tumour hypoxia induces a metabolic shift causing acidosis: A common feature in cancer. J. Cell. Mol. Med. 14 (2010) 771-794.

[19] A. Chauvière, T. Hillen, L. Preziosi, Modeling cell movement in anisotropic and heterogeneous network tissues. Networks \& Heterogeneous Media 2 (2007) 333-357.

[20] L. Chen, K. Painter, C. Surulescu, A. Zhigun, Mathematical models for cell migration: a non-local perspective. Phil. Trans. R. Soc. B (in press) arXiv:1911.05200v1. 
[21] O. Clatz, M. Sermesant, P. Bondiau, H. Delignette, S. Warfield, G. Malandain, N. Ayache, Realistic simulation of the 3D growth of brain tumors in MRI images coupling diffusion with biomechanical deformation. IEEE Trans. Med. Imaging, 24 (2005), 1334-1346.

[22] M. Conte, L. Gerardo-Giorda, M. Groppi, Glioma invasion and its interplay with the nervous tissue: a multiscale model. J. Theor. Biol. 486 (2020): 110088

[23] G. Corbin, A. Hunt, A. Klar, F. Schneider, C. Surulescu, Higher-order models for glioma invasion: from a two-scale description to effective equations for mass density and momentum, Math. Mod. Meth. Appl. Sci. 28 (2018) 1771-1800.

[24] M. Descoteaux, High angular resolution diffusion MRI: from local estimation to segmentation and tractography. Ph.D. Thesis, Université Nice-Sophia Antipolis, 2008.

[25] C. Engwer, T. Hillen, M. Knappitsch, C. Surulescu, Glioma Follow White Matter Tracts; a Multiscale DTI-based Model, J. of Math. Biol. 71 (2015) 551-582.

[26] C. Engwer, A. Hunt, C. Surulescu, Effective equations for anisotropic glioma spread with proliferation: a multiscale approach, IMA J. Mathematical Medicine \& Biology 33 (2016) $435-459$.

[27] C. Engwer, M. Knappitsch, C. Surulescu, A multiscale model for glioma spread including cell-tissue interactions and proliferation, Math. Biosc. Eng. 13 (2016) 443-460.

[28] R. Erban, H.G. Othmer, From Individual to Collective Behavior in Bacterial Chemotaxis, SIAM J. Appl. Math. 65 (2004) 361-391.

[29] V. Estrella, T. Chen, M. Lloyd, J. Wojtkowiak, H. H. Cornnell, A. Ibrahim-Hashim, K. Bailey, Y. Balagurunathan, J. M. Rothberg, B. F. Sloane, J. Johnson, R. A. Gatenby, R. J. Gillies, Acidity generated by the tumor microenvironment drives local invasion. Cancer Res, 73 (2013) 1524-1535.

[30] P.T. Fletcher, S. Joshi, Riemannian geometry for the statistical analysis of diffusion tensor data. Signal Processing 87 (2007) 250-262.

[31] H.B. Frieboes, J.S. Lowengrub, S. Wise, X. Zheng, P. Macklin, E. Bearer, V. Cristini, Computer Simulation of Glioma Growth and Morphology, Neuroimage 37 (2007), S59S70.

[32] A. Giese, L. Kluwe, H. Meissner, M.E. Berens, M. Westphal, Migration of human glioma cells on myelin, Neurosurgery 38 (1996), 755-764.

[33] A. Giese, M. Westphal, Glioma invasion in the central nervous system, Neurosurgery, 39 (1996) 235-252.

[34] L. González-Méndez, A. C. Gradilla, I. Guerrero, The cytoneme connection: direct longdistance signal transfer during development. Development 146(9), dev174607 (2019).

[35] M.A. Hammoud, R. Sawaya, W. Shi, P.F. Thall, N.E. Leeds: Prognostic significance of preoperative MRI scans in glioblastoma multiforme, J. Neurooncol. 27 (1996) 65-73.

[36] H. Hatzikirou, A. Deutsch: Cellular automata as microscopic models of cell migration in heterogeneous environments, Curr. Top. Dev. Biol. 81 (2008) 401-434.

[37] C. Henker, T. Kriesen, Ä. Glass, B. Schneider, J. Piek, Volumetric quantification of glioblastoma: experiences with different measurement techniques and impact on survival. J Neurooncol 135 (2017) 391-402.

[38] T. Hillen, $M^{5}$ mesoscopic and macroscopic models for mesenchymal motion, J. Math. Biol. 53 (2006) 585-616.

[39] T. Hillen, K. Painter, Transport and anisotropic diffusion models for movement in oriented habitats, Dispersal, Individual Movement and Spatial Ecology (M. Lewis, P. Maini \& S. Petrovskii eds). Lecture Notes in Mathematics, vol. 2071. Berlin, Heidelberg, New York: Springer 2013, p. 46.

[40] T. Hillen, A. Swan, The Diffusion Limit of Transport Equations in Biology. In: L. Preziosi, P. Ciarletta, T. Hillen, M. Chaplain, A. Pugliese, H. Othmer, D. Trucu (eds.), Mathematical Models and Methods for Living Systems, LNM 2167, Springer, 2016.

[41] A. Hunt, DTI-Based Multiscale Models for Glioma Invasion, PhD thesis, TU Kaiserslautern, 2017.

[42] A. Hunt, C. Surulescu, A multiscale modeling approach to glioma invasion with therapy, Vietnam J. Math. 45 (2017) 221-240. 
[43] A. Jbabdi, E. Mandonnet, H. Duffau, L. Capelle, K. Swanson, M. Pelegrini-Issac, R. Guillevin, H. Benali, Simulation of anisotropic growth of low-grade gliomas using diffusion tensor imaging. Mang. Res. Med., 54 (2005) 616-624.

[44] B. Jellison, A. Field, J. Medow, M. Lazar, M. Salamat, A. Alexander, Diffusion tensor imaging of cerebral white matter: a pictorial review of physics, fiber tract anatomy, and tumor imaging patterns. Am. J. Neuroradiol., 25 (2004) 356-369.

[45] A.R. Kansal, S. Torquato, G.R. Harsh IV, E.A. Chiocca, T.S. Deisbrock, Cellular automaton of idealized brain tumor growth dynamics, BioSystems 55 (2000) 119-127.

[46] A.R. Kansal, S. Torquato, G.R. Harsh IV, E.A. Chiocca, T.S. Deisbrock, Simulated Brain Tumor Growth Dynamics Using a Three-Dimensional Cellular Automaton, J. Theor. Biol. 203 (2000) 367-382.

[47] Y. Kato, S. Ozawa, C. Miyamoto, Y. Maehata, A. Suzuki, T. Maeda, Y. Baba, Acidic extracellular microenvironment and cancer. Cancer Cell Int. 13 (2013) 8 pp.

[48] J. Kelkel, C. Surulescu, On some models for cancer cell migration through tissue, Math. Biosci. Engrg. 8 (2011), 575-589.

[49] J. Kelkel, C. Surulescu, A multiscale approach to cell migration in tissue networks, Math. Mod. Meth. Appl. Sci. 22 (2013), 1150017 (25 p.).

[50] P.T. Kim, D. Richards, Deconvolution density estimation on the space of positive definite symmetric matrices. In Nonparametric Statistics and Mixture Models: A Festschrift in Honor of Thomas P Hettmansperger (pp. 147-168). World Scientific Publishing Co., 2011.

[51] P. Kleihues, F. Soylemezoglu, B. Schäuble, B.W. Scheithauer, P.C. Burger, Histopathology, classification and grading of gliomas. Glia 5 (1995) 211-221.

[52] N. Kolbe, N. Sfakianakis, C. Stinner, C. Surulescu, J. Lenz, Modeling multiple taxis: tumor invasion with phenotypic heterogeneity, haptotaxis, and unilateral interspecies repellence, arXiv:2005.01444v1.

[53] E. Konukoglu, O. Clatz, P. Bondiau, H. Delignette, N. Ayache, Extrapolation glioma invasion margin in brain magnetic resonance images: suggesting new irradiation margins. Med. Image Anal., 14 (2010) 111-125.

[54] T.B. Kornberg, L. Gilboa, Developmental biology: Nanotubes in the niche. Nature 523 (2015) 292-293.

[55] J.M. Kros, Grading of Gliomas: The Road From Eminence to Evidence, J. Neuropathol. Exp. Neurol. 70 (2011) 101-109.

[56] L.Y. Kucheryavykh, Y.V. Kucheryavykh, K. Rolon-Reyes, S.N. Skatchkov, M.J. Eaton, L.A. Cubano, M. Inyushin, Visualization of implanted GL261 glioma cells in living mouse brain slices using fluorescent 4-(4-(dimethylamino)-styryl)-N-methylpyridinium iodide $(\mathrm{ASP}+)$. BioTechniques, 53 (2012) 305-309.

[57] P. Kumar, C. Surulescu, Modeling glioma pseudopalisade patterning: a multiscale approach, in preparation.

[58] D.A. Lauffenburger, J.L. Lindermann, Receptors. Models for binding, trafficking and signaling. Oxford University Press, 1993.

[59] M. Lemou and L. Mieussens, A new asymptotic preserving scheme based on micro-macro formulation for linear kinetic equations in the diffusion limit. SIAM J. Sci. Comput. 31 (2008) 334-368.

[60] D.R. Lide (ed). CRC handbook of chemistry and physics, vol. 85. CRC Press, 2004.

[61] T. Lorenz, C. Surulescu, On a class of multiscale cancer cell migration models: wellposedness in less regular function spaces, Math. Mod. Meth. Appl. Sci. 24 (2014) 23832436.

[62] D. Louis: Molecular Pathology of Malignant Gliomas, Annu. Rev. Pathol. 1 (2006) 97-117.

[63] N. Loy, L. Preziosi, Kinetic models with non-local sensing determining cell polarization and speed according to independent cues, J. Math. Biol. 80 (2020) 373-421.

[64] N.K. Martin, E.A. Gaffney, R.A. Gatenby, R.J. Gillies, I.F. Robey, and P.K. Maini. A mathematical model of tumour and blood pHe regulation: The buffering system. Mathematical Biosciences, 230 (2011) 1-11. 
[65] G.R. Martin and R.K. Jain. Noninvasive measurement of interstitial ph profiles in normal and neoplastic tissue using fluorescence ratio imaging microscopy. Cancer Research, 54 (1994) 5670-5674.

[66] J. Mercapide, R. Lopez De Cicco, J.S. Castresana, and A.J.P. Klein-Szanto. Stromelysin$1 /$ matrix metalloproteinase-3 (MMP-3) expression accounts for invasive properties of human astrocytoma cell lines. International Journal of Cancer, 106 (2003) 676-682.

[67] R. Milo and R. Phillips, Cell biology by the numbers, Garland Science, 2015.

[68] J. Nieto and L. Urrutia: A multiscale model of cell mobility: From a kinetic to a hydrodynamic description, J. Math. Anal. Appl. 433 (2016) 1055-1071.

[69] T. Ohtsubo, X. Wang, A. Takahashi, K. Ohnishi, H. Saito, et al. p53-dependent induction of WAF1 by a low-pH culture condition in human glioblastoma cells. Cancer Res. 57 (1997) 3910-3913.

[70] H.G. Othmer, T. Hillen, The diffusion limit of transport equations II: Chemotaxis equations. SIAM Journal on Applied Mathematics, 62 (2003) 1220-1250.

[71] K. Painter, Modelling cell migration strategies in the extracellularmatrix. J. Math. Biol. 58 (2009) 511-543.

[72] K. Painter, T. Hillen, Mathematical modelling of glioma growth: the use of diffusion tensor imaging (DTI) data to predict the anisotropic pathways of cancer invasion, J. Theor. Biol. 323 (2013) 25-39.

[73] B. Perthame, M. Tang, N. Vauchelet, Derivation of the bacterial run-and-tumble kinetic equation from a model with biochemical pathway, J. Math. Biol. 73 (2016), pp.1161-1178.

[74] R.G. Plaza, Derivation of a bacterial nutrient-taxis system with doubly degenerate crossdiffusion as the parabolic limit of a velocity-jump process. J. Math. Biol. 78 (2019) 16811711.

[75] G.C. Pomraning, The Equations of Radiation Hydrodynamics. Dover books on physics (2005)

[76] M. Portela, et al., Glioblastoma cells vampirize WNT from neurons and trigger a JNK/MMP signaling loop that enhances glioblastoma progression and neurodegeneration. PLoS Biol. 17(12) (2019).

[77] D. Poyato, J. Soler, Euler-type equations and commutators in singular and hyperbolic limits of kinetic Cucker-Smale models, Math. Mod. Meth. Appl. Sci, 27(6) (2017), pp. 1089-1152.

[78] S.M. Raza, G.N. Fuller, C.H. Rhee, S. Huang, K. Hess, W. Zhang, R. Sawaya, Identification of Necrosis-Associated Genes in Glioblastoma by cDNA Microarray Analysis, Clin. Canc. Res. 10 (2004) 212-221.

[79] C. Stock, A. Schwab, Protons make tumor cells move like clockwork. Pflugers Arch. 458 (2009) 981-992.

[80] P.C. Sundgren, Q. Dong, D. Gomez-Hassan, S.K. Mukherji, P. Maly, R. Welsh, Diffusion tensor imaging of the brain: Review of clinical applications, Neurocarciology, 46 (2004) 339-350.

[81] K.R. Swanson, R.C. Rockne, J. Claridge, M.A. Chaplain, E.C. Alvord, and A.R.A. Anderson. Quantifying the role of angiogenesis in malignant progression of gliomas: In silico modeling integrates imaging and histology. Cancer Research, 71 (2011) 7366-7375.

[82] M.L. Tanaka, W. Debinski, I.K. Puri, Hybrid mathematical model of glioma progression, Cell Prolif. 42 (2009) 637-646.

[83] van der Vorst, Bi-cgstab: A fast and smoothly converging variant of bi-cg for the solution of nonsymmetric linear systems. SIAM Journal on Scientific and Statistical Computing 13 (1992) 631-644.

[84] P. Vaupel, F. Kallinowski, P. Okunieff: Blood flow, oxygen and nutrient supply, and metabolic microenvironment of human tumors: A review. Cancer Res. 49 (1989) 64496465 .

[85] C. Wang, J. Rockhill, M. Mrugala, D. Peacock, A. Lai, K. Jusenius, J. Wardlaw, T. Cloughesy, A. Spence, R. Rockne, E. Alvord Jr., K. Swanson, Prognostic significance of growth kinetics in newly diagnosed glioblastomas revealed by combining serial imaging with a novel biomathematical model. Cancer Res., 69 (2009) 9133-9140. 
[86] B.A. Webb, M. Chimenti, M.P. Jacobson, D.L. Barber, Dysregulated pH: A perfect storm for cancer progression. Nature Rev. Cancer 11 (2011) 671-677.

[87] M. Winkler, Singular structure formation in a degenerate haptotaxis model involving myopic diffusion. J. Math. Pures Appl. 112 (2018) 118-169.

[88] M. Winkler, C. Surulescu, Global weak solutions to a strongly degenerate haptotaxis model. Commun. Math. Sci. 15 (2017) 1581-1616.

[89] F. J. Wippold, M. Lämmle, F. Anatelli, J. Lennerz, A. Perry, Neuropathology for the neuroradiologist: palisades and pseudopalisades. American Journal of Neuroradiology 27 (2006) 2037-2041.

[90] R.K.W. Wong, T.C.M. Lee, D. Paul, J. Peng, Fiber direction estimation, smoothing, and tracking in diffusion MRI. Ann. appl. Stat. 10 (2016) 1137-1156.

[91] M. Wrensch, Y. Minn, T. Chew, M. Bondy, M. S. Berger, Epidemiology of primary brain tumors: Current concepts and review of the literature, Neuro-Oncology, 4 (2002) 278-299.

[92] C. Xue, H.G. Othmer, Multiscale models of taxis-driven patterning in bacterial populations, SIAM J. Appl. Math. 70 (2009) 133-169.

[93] L. Zhang, B. Jiang, Y. Wu, C. Strouthos, P. Zhe Sun, J. Su, X. Zhou, Developing a multiscale, multi-resolution agent- based brain tumor model by graphics processing units, Theor. Biol. Medical Mod. 8:46 (2011). 